\title{
Analytical solution for free vibrations of rotating cylindrical shells having free boundary conditions
}

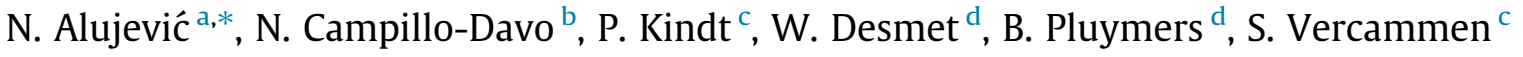 \\ ${ }^{a}$ University of Zagreb, Faculty of Mechanical Engineering and Naval Architecture, Ivana Lučića 5, 10000 Zagreb, Croatia \\ ${ }^{\mathrm{b}}$ Universidad Miguel Hernandez de Elche, Dpto. de Ingeniería Mecánica y Energía, Área de Ingeniería Mecánica, Avda. de la Universidad, S/N. Edif. Quorum V, 03202 Elche \\ (Alicante), Spain \\ ${ }^{\mathrm{c}}$ Goodyear S.A., Innovation Center, L-7750 Colmar-Berg, Luxembourg \\ ${ }^{\mathrm{d}}$ KU Leuven, Production Engineering, Machine Design and Automation (PMA) Section, Celestijnenlaan 300b - Box 2420, 3001 Heverlee, Belgium
}

\section{A R T I C L E I N F O}

\section{Article history:}

Received 19 February 2015

Revised 28 October 2016

Accepted 3 November 2016

\section{Keywords:}

Vibration

Circular cylindrical shells

Free boundary conditions

Rotating shells

Elastic foundation

Rotating modes

Analytical methods

Natural frequencies

Mode shapes

\begin{abstract}
A B S T R A C T
In this paper free vibrations of rotating cylindrical shells with both ends free are studied. The model used also allows for considering a flexible foundation supporting the shell in the sense of a radial and circumferential distributed stiffness. Furthermore, a circumferential tension (hoop stress) which may be due to pressurisation or centrifugal forces is taken into account. Natural frequencies and mode shapes are determined exactly for both stationary shells and for shells rotating with a constant angular speed around the cylinder axis. Trigonometric functions are assumed for the circumferential mode shape profiles, and a sum of eight weighted exponential functions is assumed for the axial mode shape profiles. The functional form of the axial profiles is shown to greatly vary with the roots of a characteristic bi-quartic polynomial that occurs in the process of satisfying the equations of motion. In the previously published work it has been very often assumed that the roots are two real, two imaginary, and two pairs of complex conjugates. In the present study, a total of eight types of roots are shown to determine the whole set of mode shapes, either for stationary or for rotating shells. The results using the developed analytical model are compared with results of experimental studies and very good agreement is obtained. Also, a parametric study is carried out where effects of the elastic foundation stiffnesses and the rotation speed are examined.
\end{abstract}

(c) 2016 Elsevier Ltd. All rights reserved.

\section{Introduction}

Dynamics of shells have been an active research topic for well over a century. Some early works dating from the 19th century [1-5], were followed by the developments in the 20th century [6-19]. Many geometries occurring in various engineering structures can be seen as shells. Among these, circular cylindrical shells form a particular class. It is often the case that a cylindrical shell spins around its axis, which makes its dynamic behaviour more complex. Rotating shell structures are found in engineering applications such as rotor systems of gas turbine engines, high-speed centrifugal separators, rotating satellite structures, and automotive tires, to name a few.

Early studies on rotating cylinders include the work of Bryan [20] who studied vibrations of a rotating ring and described the travelling modes phenomenon. Di Taranto and Lessen [21], and also Srinivasan and Lauterbach [22] studied Coriolis and centrifu-

\footnotetext{
* Corresponding author.

E-mail address: neven.alujevic@mech.kuleuven.be (N. Alujević).
}

gal effects on infinitely long rotating cylindrical shells. Zohar and Aboudi [23], and also Saito and Endo [24] presented such investigations on finite long rotating cylinders. Endo et al. [25] performed an experimental study of flexural vibration of a thin rotating ring. Padovan [26] studied the free vibration of rotating cylinders subjected to pre-stress. Kim and Bolton also considered the effects of rotation on the dynamics of a circular cylindrical shell [27]. They suggested that the model may be used to predict the characteristics of a rotating tire after performing a kinematic compensation on the results of a stationary tire analysis. Huang and Soedel [28] used the nonlinear strain displacement relationships of Herrmann and Armenakas [29] and the corresponding set of equations of motion for a spinning shell in the co-rotating reference frame. The authors have solved the free and forced vibration problem assuming simply supported boundary conditions (the so-called shear diaphragm boundary conditions). This is a favourable type of a boundary condition from a mathematical point of view. This is because mode shape axial profiles do not exhibit a change of their functional form. Thus simple sine or cosine functions of the axial coordinate may be used [18,19,27-30]. The natural frequencies can be calculated as roots of a characteristic polynomial, which 
was shown to be bi-cubic if the shell does not rotate. In case the shell spins at a constant speed, also the odd coefficients of the polynomial occur. Thus in case of a non-rotating cylindrical shell this bi-cubic polynomial has three pairs of roots where each pair consists of a positive and a negative natural frequency having the same absolute value. Physically this underlies the existence of the backward and forward rotating modes that superimpose into "regular" vibration modes in case the cylinder is stationary. With spinning cylinders the six natural frequencies have distinct absolute values, and thus the rotating modes occur [28]. The three pairs of positive and negative frequencies correspond to three types of modes, which could be named bending modes, longitudinal modes, and shear modes. This classification is based on whether the radial, axial, or circumferential displacement component is the most prominent in a particular vibration pattern.

In general, short expressions for calculating natural frequencies of either rotating or stationary cylindrical shells are not possible if no further simplifications or assumptions are made to reduce the order of the characteristic polynomial [17,19,31-33]. For example, the Donnel-Mushtari-Vlasov equation can yield a reasonably short closed form expression for natural frequencies of a non-rotating cylindrical shell provided that simply supported boundary conditions are assumed [19].

However, in case of other boundary conditions the situation complicates. Consequently a number of studies have also been dedicated to vibration of cylindrical shells with other types of simple boundary conditions [34-37]. For example, Chung expressed the displacements as product of Fourier series for the axial modal displacements and trigonometric functions for the circumferential modal displacements. The author used Stokes' transformation to obtain expressions for derivatives of the Fourier series [37]. Boundary conditions such as free-free, clamped-free and clampedclamped are considered in the study. This methodology has been recently extended by Sun et al. [38] to rotating cylindrical shells including the effects of centrifugal and Coriolis forces and the initial hoop tension. Alternatively, the Rayleigh-Ritz method can be employed to derive the frequency equations of rotating cylinders. Utilising the Rayleigh-Ritz method, Sun et al. [39] took the characteristic orthogonal polynomial series as the admissible functions with classical homogeneous boundary conditions, or with more general boundary conditions, by utilising artificial springs to simulate the elastic constraints imposed.

An exact approach to deal with other types of boundary conditions has been used by Warburton [36]. He analysed the free vibration problem using Flügge equations and considered either both ends clamped or both ends free of a non-rotating cylindrical shell.
A number of mode shapes and natural frequencies were calculated in [36] by assuming identical boundary conditions at the two ends of the shell, and analysing separately symmetric and antisymmetric modes. The author considered the case where the roots of the characteristic polynomial are of a particular form: two real, two imaginary and four complex.

A complete analytical solution for free vibrations of a circular cylindrical shell of finite length, supported by an elastic foundation, having both ends free, either stationary or rotating, is given in this paper. The equations of motion are based on the straindisplacement relationships of Hermann and Armenakas [29]. It is shown that it is necessary to consider eight types of roots of the characteristic polynomial in order to derive eight types of mode shapes. All types of modes may occur with both non-rotating and spinning shells having free ends. For each mode shape type the free-free boundary conditions are satisfied exactly. In order for the boundary conditions to be satisfied, the determinant of the boundary condition matrix must vanish. This fact is used to determine the natural frequencies of both stationary and rotating shells.

The paper is structured into five sections. The mathematical model is developed in the second section. The free vibrations of an example rotating shell are discussed in the third section. The third section also contains a comparison of the analytical results to results of different experimental studies. The fourth section is dedicated to a parametric study where the effect on the natural frequencies of different parameters of the model is studied. Appendix to the paper contains various coefficients needed to shorten the main expressions in the paper expressed as a function of the material and geometrical shell parameters.

\section{Mathematical model}

The rotating cylindrical shell is shown schematically in Fig. 1.

Assuming the free vibration problem the equations of motion are $[28,29]$ :

$$
\left[\begin{array}{ccc}
\mathrm{L}_{x, u}+\rho h \frac{\partial^{2}}{\partial t^{2}} & \mathrm{~L}_{x, v} & \mathrm{~L}_{x, w} \\
\mathrm{~L}_{\phi, u} & \mathrm{~L}_{\phi, v}+\rho h\left(\frac{\partial^{2}}{\partial t^{2}}-\Omega^{2}\right) & \mathrm{L}_{\phi, w}+2 \rho h \Omega \frac{\partial}{\partial t} \\
\mathrm{~L}_{z, u} & \mathrm{~L}_{z, v}-2 \rho h \Omega \frac{\partial}{\partial t} & \mathrm{~L}_{z, w}+\rho h\left(\frac{\partial^{2}}{\partial t^{2}}-\Omega^{2}\right)
\end{array}\right]\left\{\begin{array}{c}
u \\
v \\
w
\end{array}\right\}=\mathbf{0} .
$$

The linear operators $\mathrm{L}_{x, u}, \mathrm{~L}_{x, v}, \mathrm{~L}_{x, w}, \mathrm{~L}_{\phi, u}, \mathrm{~L}_{\phi, v}, \mathrm{~L}_{\phi, w}$ and $\mathrm{L}_{z, u}, \mathrm{~L}_{z, v}, \mathrm{~L}_{z, w}$ are:

$\mathrm{L}_{x, u}=\frac{(\mu-1) K-2 N_{\phi, i}}{2 a^{2}} \frac{\partial^{2}}{\partial \phi^{2}}-\left(K+N_{x, i}\right) \frac{\partial^{2}}{\partial x^{2}}$,
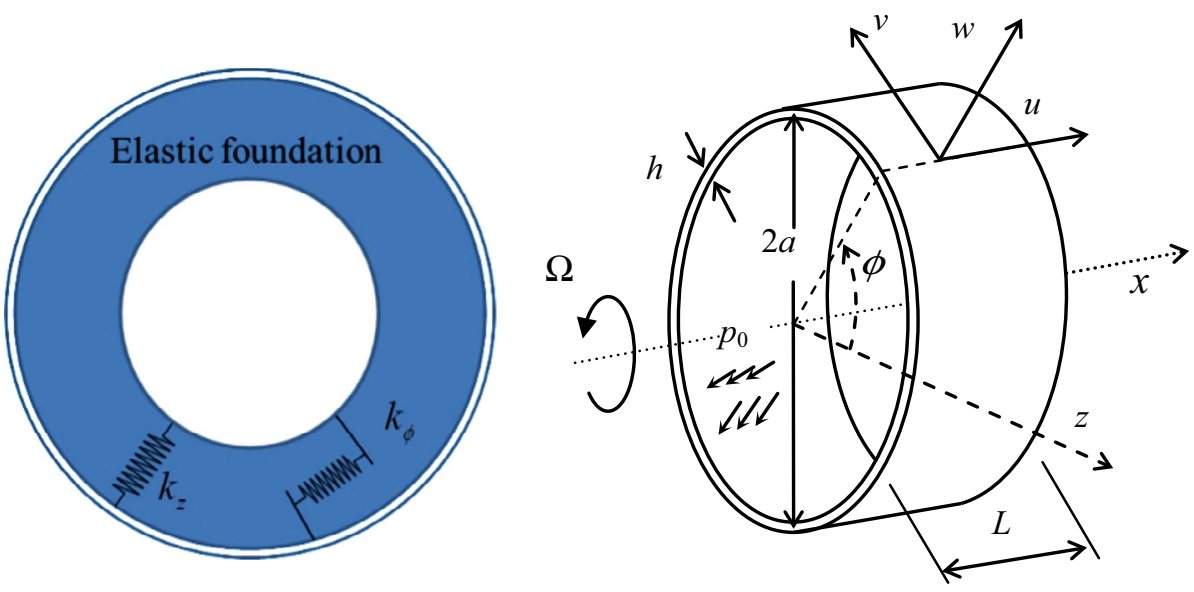

Fig. 1. The rotating cylindrical shell. 
$\mathrm{L}_{x, v}=-\frac{K(1+\mu)}{2 a} \frac{\partial^{2}}{\partial x \partial \phi}$

$\mathrm{L}_{x, w}=-\frac{\mu K}{a} \frac{\partial}{\partial x}$

$\mathrm{L}_{\phi, u}=-\frac{K(1+\mu)}{2 a} \frac{\partial^{2}}{\partial x \partial \phi}$,

$\mathrm{L}_{\phi, v}=\frac{(\mu-1)\left(K a^{2}+D\right)-2 N_{x, i} a^{2}}{2 a^{2}} \frac{\partial^{2}}{\partial x^{2}}-\frac{\left(\left(K+N_{\phi, i}\right) a^{2}+D\right)}{a^{4}} \frac{\partial^{2}}{\partial \phi^{2}}$

$$
+\frac{\left(k_{\phi} a^{2}+N_{\phi, i}\right)}{a^{2}} v
$$

$\mathrm{L}_{\phi, w}=\frac{D}{a^{4}} \frac{\partial^{3}}{\partial \phi^{3}}-\frac{\left(K+2 N_{\phi, i}\right) \frac{\partial}{\partial \phi}-D \frac{\partial^{3}}{\partial x^{2} \partial \phi}}{a^{2}}$

$\mathrm{L}_{z, u}=\frac{\mu K}{a} \frac{\partial}{\partial x}$,

$\mathrm{L}_{z, v}=\frac{\left(K+2 N_{\phi, i}\right) \frac{\partial}{\partial \phi}-D \frac{\partial^{3}}{\partial x^{2} \partial \phi}}{a^{2}}-\frac{D}{a^{4}} \frac{\partial^{3}}{\partial \phi^{3}}$,

$\mathrm{L}_{z, w}=\frac{D}{a^{4}} \frac{\partial^{4}}{\partial \phi^{4}}$

$$
+\frac{D\left(2 \frac{\partial^{4}}{\partial x^{2} \partial \phi^{2}}+a^{2} \frac{\partial^{4}}{\partial x^{4}}\right)-N_{\phi, i} \frac{\partial^{2}}{\partial \phi^{2}}-a^{2} N_{x, i} \frac{\partial^{2}}{\partial x^{2}}+\left(K+a^{2} k_{z}+N_{\phi, i}\right)}{a^{2}},
$$

where

$$
\begin{aligned}
& a=\text { shell radius, } \\
& h=\text { shell thickness, } \\
& L=\text { shell length, } \\
& p_{0}=\text { inflation pressure, } \\
& u=u(x, \phi, t)=\text { axial displacement, } \\
& v=v(x, \phi, t)=\text { tangential displacement, } \\
& w=w(x, \phi, t)=\text { radial displacement, } \\
& x=\text { axial coordinate, } \\
& \phi=\text { tangential coordinate, } \\
& z=\text { radial coordinate, } \\
& \mu=\text { Poisson's ratio, } \\
& E=\text { Young's modulus, } \\
& \rho=\text { mass density, } \\
& k_{\phi}=\text { elastic foundation stiffness in the tangential direction, } \\
& k_{z}=\text { elastic foundation stiffness in the radial direction, } \\
& N_{x, i}=\text { initial tension in the axial direction, } \\
& N_{\phi, i}=\text { initial tension in the tangential direction, } \\
& \Omega=\text { rotation speed, } \\
& D=\frac{E h^{3}}{12\left(1-\mu^{2}\right)}=\text { bending stiffness, and } \\
& K=\frac{E h}{1-\mu^{2}}=\text { membrane stiffness. }
\end{aligned}
$$

The initial tension in the tangential direction is given by [28]:

$$
N_{\phi, i}=\rho h a^{2} \Omega^{2}+a p_{0},
$$

where the first term is due to centrifugal forces and the second term is due to the initial inflation pressure of the shell. Substituting:

$u=U_{0} \mathrm{e}^{\frac{\alpha x}{a}} \cos \left(n \phi+\omega_{m, n} t\right)$,

$v=V_{0} \mathrm{e}^{\frac{\alpha x}{a}} \sin \left(n \phi+\omega_{m, n} t\right)$,

$w=W_{0} \mathrm{e}^{\frac{\alpha x}{a}} \cos \left(n \phi+\omega_{m, n} t\right)$ where $n$ is the circumferential mode number, and $m$ is the axial mode number, into the equations of motion (1), yields:

$$
\left[\begin{array}{ccc}
k_{1,1, A}+k_{1,1, B} \alpha^{2} & k_{1,2, B} \alpha & k_{1,3, B} \alpha \\
\text { symm. } & k_{2,2, A}+k_{2,2, B} \alpha^{2} & k_{2,3, A}+k_{2,3, B} \alpha^{2} \\
\text { symm. } & \text { symm. } & k_{3,3, A}+k_{3,3, B} \alpha^{2}+k_{3,3, C} \alpha^{4}
\end{array}\right]\left\{\begin{array}{c}
U_{0} \\
V_{0} \\
W_{0}
\end{array}\right\}=\mathbf{0} \text {, }
$$

where

$k_{1,1, A}=\rho h\left(\omega^{2}-n^{2} \Omega^{2}\right)-\frac{n^{2} p_{0}}{a}+\frac{n^{2} K(\mu-1)}{2 a^{2}}$,

$k_{1,1, B}=\frac{K+N_{x, i}}{a^{2}}$,

$k_{1,2, B}=\frac{K(1+\mu) n}{2 a^{2}}$,

$k_{1,3, B}=\frac{\mu K}{a^{2}}$,

$k_{2,2, A}=\rho h\left(n^{2} \Omega^{2}-\omega^{2}\right)+k_{\phi}+\frac{p_{0}\left(1+n^{2}\right)}{a}+\frac{n^{2} K}{a^{2}}+\frac{D n^{2}}{a^{4}}$,

$k_{2,2, B}=\frac{K(\mu-1)-2 N_{x, i}}{2 a^{2}}+\frac{D(\mu-1)}{2 a^{4}}$,

$k_{2,3, A}=2 h \rho\left(n \Omega^{2}-\omega \Omega\right)+\frac{2 n p_{0}}{a}+\frac{n K}{a^{2}}+\frac{D n^{3}}{a^{4}}$,

$k_{2,3, B}=-\frac{n D}{a^{4}}$,

$k_{3,3, A}=\rho h\left(n^{2} \Omega^{2}-\omega^{2}\right)+k_{z}+\frac{p_{0}\left(1+n^{2}\right)}{a}+\frac{K}{a^{2}}+\frac{D n^{4}}{a^{4}}$,

$k_{3,3, B}=\frac{-a^{2} N_{x, i}-2 D n^{2}}{a^{4}}$,

$k_{3,3, C}=\frac{D}{a^{4}}$.

The determinant of the matrix in Eq. (15) must vanish in order for the equations of motion to be satisfied. Setting the determinant to zero yields a biquartic polynomial in $\alpha$ :

$A_{8} \alpha^{8}+A_{6} \alpha^{6}+A_{4} \alpha^{4}+A_{2} \alpha^{2}+A_{0}=0$,

where the coefficients $A_{r}$ in function of $k_{i, j, \sim}$ are given in Table 1 below:

So there are eight roots of the polynomial and consequently the radial component of the displacement can be expressed as:

$w=W(x) \cos \left(n \phi+\omega_{m, n} t\right)$,

Table 1

The coefficients $A_{r}$.

\begin{tabular}{ll}
\hline$A_{0} \quad k_{1,1, A} k_{2,2, A} k_{3,3, A}-k_{1,1, A} k_{2,3, A}^{2}$ \\
$A_{2} \quad\left(k_{2,2, A} k_{3,3, B}+k_{2,2, B} k_{3,3, A}-2 k_{2,3, A} k_{2,3, B}\right) k_{1,1, A}+\left(k_{3,3, A} k_{1,1, B}-k_{1,3, B}^{2}\right) k_{2,2, A}+$ \\
$\quad+2 k_{1,2, B} k_{1,3, B} k_{2,3, A}-k_{1,2, B}^{2} k_{3,3, A}-k_{1,1, B} k_{2,3, A}^{2}$ \\
$A_{4} \quad\left(k_{2,2, A} k_{3,3, C}+k_{2,2, B} k_{3,3, B}-k_{2,3, B}^{2}\right) k_{1,1, A}+\left(-2 k_{2,3, A} k_{2,3, B}+k_{2,2, A} k_{3,3, B}\right.$ \\
$\left.\quad+k_{2,2, B} k_{3,3, A}\right) k_{1,1, B}--k_{1,3, B}^{2} k_{2,2, B}+2 k_{1,2, B} k_{1,3, B} k_{2,3, B}-k_{1,2, B}^{2} k_{3,3, B}$ \\
$A_{6} \quad\left(k_{2,2, A} k_{3,3, C}+k_{2,2, B} k_{3,3, B}-k_{2,3, B}^{2}\right) k_{1,1, B}$ \\
$\quad+k_{3,3, C}\left(k_{1,1, A} k_{2,2, B}-k_{1,2, B}^{2}\right)$ \\
$A_{8} \quad k_{1,1, B} k_{2,2, B} k_{3,3, C}$ \\
\hline
\end{tabular}


with

$W(x)=\sum_{r=1}^{8} B_{r} e^{\frac{\alpha_{r} x}{a}}$

where $B_{r}$, with $r=1, \ldots, 8$, are eight generally complex constants. The roots of the polynomial (27) must be calculated at this stage by assuming the frequency $\omega_{m, n}$.

\subsection{Case 1: two real, two imaginary and four complex roots}

If the roots have the form $\pm \alpha_{1}, \pm \mathrm{i} \gamma_{2}, \pm(p \pm \mathrm{i} q)$ where $\alpha_{1}, \gamma_{2}, p, q$ are real and positive numbers [40], then the radial component of the displacement is given by:

$$
\begin{aligned}
W(x)= & C_{1} \cosh \left(\frac{\alpha_{1} x}{a}\right)+C_{2} \sinh \left(\frac{\alpha_{1} x}{a}\right)+C_{3} \cos \left(\frac{\gamma_{2} x}{a}\right) \\
& +C_{4} \sin \left(\frac{\gamma_{2} x}{a}\right)+e^{\frac{p x}{a}}\left(C_{5} \cos \left(\frac{q x}{a}\right)+C_{6} \sin \left(\frac{q x}{a}\right)\right) \\
& +e^{\frac{-p x}{a}}\left(C_{7} \cos \left(\frac{q x}{a}\right)+C_{8} \sin \left(\frac{q x}{a}\right)\right),
\end{aligned}
$$

where $C_{r}$ are now real constants. From Eq. (15):

$\left(\frac{U_{0}}{W_{0}}\right)_{r}=\frac{k_{1,2} k_{2,3}-k_{1,3} k_{2,2}}{k_{1,1} k_{2,2}-k_{1,2}^{2}}$

and

$\left(\frac{V_{0}}{W_{0}}\right)_{r}=\frac{k_{1,2} k_{1,3}-k_{2,3} k_{1,1}}{k_{1,1} k_{2,2}-k_{1,2}^{2}}$

where $k_{1,1}=k_{1,1, A}+k_{1,1, B} \alpha^{2}, k_{1,2}=k_{1,2, B} \alpha, k_{1,3}=k_{1,3, B} \alpha, k_{2,2}=k_{2,2, A}+$ $k_{2,2, B} \alpha^{2}, k_{2,3}=k_{2,3, A}+k_{2,3, B} \alpha^{2}$.

Substituting for each root $\alpha_{r}$ into Eqs. (31) and (32), the expressions for $U(x)$ and $V(x)$ can be represented as:

$V(x)=d_{1} C_{1} \cosh \left(\frac{\alpha_{1} x}{a}\right)+d_{1} C_{2} \sinh \left(\frac{\alpha_{1} x}{a}\right)+d_{3} C_{3} \cos \left(\frac{\gamma_{2} x}{a}\right)+d_{3} C_{4} \sin \left(\frac{\gamma_{2} x}{a}\right)$

$+e^{\frac{p x}{a}}\left(\left(d_{5} C_{5}+d_{6} C_{6}\right) \cos \left(\frac{q x}{a}\right)+\left(d_{5} C_{6}-d_{6} C_{5}\right) \sin \left(\frac{q x}{a}\right)\right)$

$+e^{\frac{-p x}{a}}\left(\left(d_{5} C_{7}-d_{6} C_{8}\right) \cos \left(\frac{q x}{a}\right)+\left(d_{5} C_{8}+d_{6} C_{7}\right) \sin \left(\frac{q x}{a}\right)\right)$

$U(x)=d_{2} C_{2} \cosh \left(\frac{\alpha_{1} x}{a}\right)+d_{2} C_{1} \sinh \left(\frac{\alpha_{1} x}{a}\right)+d_{4} C_{4} \cos \left(\frac{\gamma_{2} x}{a}\right)-d_{4} C_{3} \sin \left(\frac{\gamma_{2} x}{a}\right)$

$+e^{\frac{p x}{a}}\left(\left(d_{7} C_{5}+d_{8} C_{6}\right) \cos \left(\frac{q x}{a}\right)+\left(d_{7} C_{6}-d_{8} C_{5}\right) \sin \left(\frac{q x}{a}\right)\right)$

$+e^{\frac{-p x}{a}}\left(\left(-d_{7} C_{7}+d_{8} C_{8}\right) \cos \left(\frac{q x}{a}\right)-\left(d_{7} C_{8}+d_{8} C_{7}\right) \sin \left(\frac{q x}{a}\right)\right)$

The constants $d_{r}$ can now be calculated using Eqs. (31) and (32) as:

$d_{1}=\left(V_{0} / W_{0}\right)_{r}$ with $\alpha_{r}=\alpha_{1}$,

$d_{2}=\left(U_{0} / W_{0}\right)_{r}$ with $\alpha_{r}=\alpha_{1}$,

$d_{3}=\left(V_{0} / W_{0}\right)_{r}$ with $\alpha_{r}=\mathrm{i} \gamma_{2}$,

$d_{4}=\mathfrak{J}\left(U_{0} / W_{0}\right)_{r}$ with $\alpha_{r}=\mathrm{i} \gamma_{2}$,

$d_{5}=\mathfrak{R}\left(V_{0} / W_{0}\right)_{r}$ with $\alpha_{r}=p+\mathrm{i} q$,

$d_{6}=\mathfrak{J}\left(V_{0} / W_{0}\right)_{r}$ with $\alpha_{r}=p+\mathrm{i} q$,

$d_{7}=\mathfrak{R}\left(U_{0} / W_{0}\right)_{r}$ with $\alpha_{r}=p+\mathrm{i} q$,

$d_{8}=\mathfrak{J}\left(U_{0} / W_{0}\right)_{r}$ with $\alpha_{r}=p+\mathrm{i} q$

where $\mathfrak{R}$ stands for the real and $\mathfrak{\Im}$ stands for the imaginary part of the complex number.

At each end of the shell there are five resultant forces as shown in Fig. 2, where $N_{x}$ is the axial membrane force, $Q_{x, z}$ is the radial membrane force, $N_{x, \phi}$ is the circumferential membrane force, $M_{x, \phi}$ is the twisting moment, and $M_{x}$ is the bending moment.

The equations of motion can only accommodate for four boundary conditions per an end of the shell $[2,19]$. Thus the Kirchhoff effective shear stress resultant of the first kind, $V_{x z}$, and the Kirchhoff effective shear stress resultant of the second kind, $T_{x \phi}$,

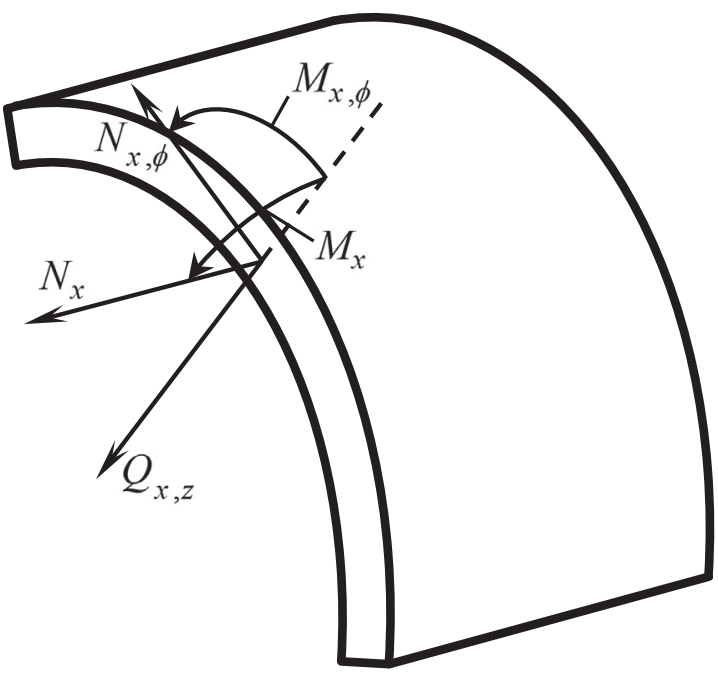

Fig. 2. The five boundary force resultants.

should be used that combine $Q_{x, z}$ and $M_{x, \phi}$, as well as $N_{x, \phi}$ and $M_{x, \phi}$, respectively $[2,18,19]$.

The relations are $[2,18,19]$ :

$V_{x z}=Q_{x z}+\frac{1}{a} \frac{\partial M_{x \phi}}{\partial \phi}$

and

$T_{x \phi}=N_{x \phi}+\frac{1}{a} M_{x \phi}$

Thus, the four boundary conditions for a shell with both ends free are:

$N_{x}=0 ; \Rightarrow a \frac{\partial u}{\partial x}+\mu w+\mu \frac{\partial v}{\partial \phi}=0$,

$M_{x}=0 ; \Rightarrow a^{2} \frac{\partial^{2} w}{\partial x^{2}}+\mu\left(\frac{\partial^{2} w}{\partial \phi^{2}}-\frac{\partial v}{\partial \phi}\right)=0$

$V_{x z}=0 ; \Rightarrow(2-\mu) \frac{\partial^{3} w}{\partial x \partial \phi^{2}}-\frac{\partial^{2} v}{\partial x \partial \phi}+a^{2} \frac{\partial^{3} w}{\partial x^{3}}=0$,

$T_{x \phi}=0 ; \Rightarrow-2 h^{2} \frac{\partial^{2} w}{\partial x \partial \phi}+\left(12 a^{2}+h^{2}\right) \frac{\partial v}{\partial x}+12 a \frac{\partial u}{\partial \phi}=0$.

Since the boundary conditions are the same at the two ends, the modes can be either symmetric or anti-symmetric; in other words, they cannot be asymmetric. Consequently the boundary conditions can be separately satisfied for symmetric and anti-symmetric modes by assuming the origin in the middle section of the cylinder and the boundaries at $x= \pm L / 2$. Here the radial and the circumferential displacement components share the symmetry or antisymmetry properties. The axial displacement component mathematical symmetry or anti-symmetry is opposite to that of the radial and circumferential displacement components but the physical symmetry is also the same. In case the modes are symmetric, the expression for the radial component of the displacement can be shortened by using $e^{ \pm p x / a}=\cosh (p x / a) \pm \sinh (p x / a)$, and making sure that the anti-symmetric terms in Eq. (30) vanish. Then it must be $C_{2}=C_{4}=0, C_{5}=C_{7}$, and $C_{6}=-C_{8}$, so that Eqs. (30), (33) and (34) reduce to:

$$
\begin{aligned}
W(x)= & C_{1} \cosh \left(\frac{\alpha_{1} x}{a}\right)+C_{3} \cos \left(\frac{\gamma_{2} x}{a}\right)+F_{1} \cosh \left(\frac{p x}{a}\right) \cos \left(\frac{q x}{a}\right) \\
& +F_{2} \sinh \left(\frac{p x}{a}\right) \sin \left(\frac{q x}{a}\right),
\end{aligned}
$$




$$
\begin{aligned}
V(x)= & d_{1} C_{1} \cosh \left(\frac{\alpha_{1} x}{a}\right)+d_{3} C_{3} \cos \left(\frac{\gamma_{2} x}{a}\right)+\left(d_{5} F_{1}+d_{6} F_{2}\right) \cosh \left(\frac{p x}{a}\right) \cos \left(\frac{q x}{a}\right), \\
& +\left(d_{5} F_{2}-d_{6} F_{1}\right) \sinh \left(\frac{p x}{a}\right) \sin \left(\frac{q x}{a}\right)
\end{aligned}
$$

$$
\begin{aligned}
U(x)= & d_{2} C_{1} \sinh \left(\frac{\alpha_{1} x}{a}\right)-d_{4} C_{3} \sin \left(\frac{\gamma_{2} x}{a}\right)+\left(d_{7} F_{1}+d_{8} F_{2}\right) \sinh \left(\frac{p x}{a}\right) \cos \left(\frac{q x}{a}\right) \\
& +\left(d_{7} F_{2}-d_{8} F_{1}\right) \cosh \left(\frac{p x}{a}\right) \sin \left(\frac{q x}{a}\right)
\end{aligned}
$$

Substituting Eq. (42) into (28) for the radial displacement component, and also Eqs. (43) and (44) into analogue expressions for tangential and axial displacement components gives a set of displacement components $u, v$ and $w$. These can be substituted into Eqs. (38)-(41), which after assuming $x=L / 2$ and putting into a matrix form gives: indicating that the forward and backward rotating modes are now characterised by different rotation speeds. Thus for rotating shells negative frequencies must be assumed in order to account for the forward rotating modes.

In case of anti-symmetric modes it must be $C_{1}=C_{3}=0$, $C_{5}=-C_{7}$, and $C_{6}=C_{8}$ so that:

$$
\begin{aligned}
W(x)= & C_{2} \sinh \left(\frac{\alpha_{1} x}{a}\right)+C_{4} \sin \left(\frac{\gamma_{2} x}{a}\right)+F_{3} \sinh \left(\frac{p x}{a}\right) \cos \left(\frac{q x}{a}\right) \\
& +F_{4} \cosh \left(\frac{p x}{a}\right) \sin \left(\frac{q x}{a}\right),
\end{aligned}
$$

with similar expressions for $V(x)$ and $U(x)$. After recalculating the determinant for the anti-symmetric modes an expression analogue to Eq. (46) results, which can be obtained by substituting

$$
\left[\begin{array}{ccccc}
t_{1,1} \cosh \left(\theta_{1}\right) & t_{1,2} \cos \left(\theta_{2}\right) & t_{1,3, A} \cos \left(\theta_{4}\right) \cosh \left(\theta_{3}\right)+ & t_{1,4, A} \cos \left(\theta_{4}\right) \cosh \left(\theta_{3}\right)+ \\
& & +t_{1,3, B} \sin \left(\theta_{4}\right) \sinh \left(\theta_{3}\right) & +t_{1,4, B} \sin \left(\theta_{4}\right) \sinh \left(\theta_{3}\right) \\
t_{2,1} \cosh \left(\theta_{1}\right) & t_{2,2} \cos \left(\theta_{2}\right) & t_{2,3, A} \cos \left(\theta_{4}\right) \cosh \left(\theta_{3}\right)+ & t_{2,4, A} \cos \left(\theta_{4}\right) \cosh \left(\theta_{3}\right)+ \\
& & +t_{2,3, B} \sin \left(\theta_{4}\right) \sinh \left(\theta_{3}\right) & +t_{2,4, B} \sin \left(\theta_{4}\right) \sinh \left(\theta_{3}\right) \\
& & t_{3,3, A} \sin \left(\theta_{4}\right) \cosh \left(\theta_{3}\right)+ & t_{3,4, A} \sin \left(\theta_{4}\right) \cosh \left(\theta_{3}\right)+ \\
t_{3,1} \sinh \left(\theta_{1}\right) & t_{3,2} \sin \left(\theta_{2}\right) & +t_{3,3, B} \cos \left(\theta_{4}\right) \sinh \left(\theta_{3}\right) & +t_{3,4, B} \cos \left(\theta_{4}\right) \sinh \left(\theta_{3}\right) \\
& & t_{4,3, A} \sin \left(\theta_{4}\right) \cosh \left(\theta_{3}\right)+ & t_{4,4, A} \sin \left(\theta_{4}\right) \cosh \left(\theta_{3}\right)+ \\
t_{4,1} \sinh \left(\theta_{1}\right) & t_{4,2} \sin \left(\theta_{2}\right) & +t_{4,3, B} \cos \left(\theta_{4}\right) \sinh \left(\theta_{3}\right) & +t_{4,4, B} \cos \left(\theta_{4}\right) \sinh \left(\theta_{3}\right)
\end{array}\right]\left\{\begin{array}{l}
C_{1} \\
C_{3} \\
F_{1} \\
F_{2}
\end{array}\right\}=0,
$$

where $\theta_{1}=\frac{\alpha_{1} L}{2 a}, \theta_{2}=\frac{\gamma_{2} L}{2 a}, \theta_{3}=\frac{p L}{2 a}$ and $\theta_{4}=\frac{q L}{2 a}$. The coefficients $t_{r, s, \sim}$ depend on the roots $\alpha_{1}, \gamma_{2}, p, q$, the constants $d_{r}, n$ and $\mu$, and are listed in the Appendix to the paper. In order to satisfy the boundary conditions, the determinant of the matrix in Eq. (45) must vanish. The determinant is a function of the length $L$ which oscillates around zero and diverges very quickly with an increase in length, covering a very large range of several tens of orders of magnitude due to the hyperbolic sine and cosine functions involved. However, the interest here is to have the determinant equal to zero. It is thus useful to divide the determinant by $\sinh \left(\frac{p L}{2 a}\right) \sinh \left(\frac{\alpha_{1} L}{2 a}\right) \cosh \left(\frac{p L}{2 a}\right)$ for better numerical behaviour. Such normalised determinant function still oscillates with the length $L$ and has the same zeroes as the original determinant; however it is much easier to deal with from a numerical point of view since it is now in terms of hyperbolic tangent and cotangent functions. The condition on the normalised determinant is given by: $\tanh \left(\theta_{1}\right) \leftrightarrow \operatorname{coth}\left(\theta_{1}\right), \tanh \left(\theta_{3}\right) \leftrightarrow \operatorname{coth}\left(\theta_{3}\right), \cos \left(\theta_{2}\right) \rightarrow \sin \left(\theta_{2}\right)$, and $\sin \left(\theta_{2}\right) \rightarrow-\cos \left(\theta_{2}\right)$ into Eq. (46). Then the zeroes represent the length of the shell whose $m, n$ mode resonates at the assumed frequency $\omega_{m, n}$, where the lowest zero is for $m=0$ anti-symmetric mode (Love-type mode with one nodal cross section), the next one is for $m=2$ anti-symmetric mode, etc. In order to calculate natural frequencies for a shell of given length it is necessary to iterate until the length resulting from finding a zero of the determinant (46) matches the desired length of the shell to an acceptable precision. An alternative approach utilises a detection of two frequencies between which the determinant (46) changes sign for the shell of a given length.

However, the roots of type $\pm \alpha_{1}, \pm \mathrm{i} \gamma_{2}, \pm(p \pm \mathrm{i} q)$ and the resulting expressions for the mode shape axial profiles do not cover for all types of modes. For example, the symmetric Rayleigh-type modes $(m=-1)$ do require a separate form of roots, according to findings

$\left(\left(\tanh \left(\theta_{3}\right) b_{1} b_{2} \cos \left(\theta_{2}\right)+\sin \left(\theta_{2}\right) b_{3}\right) \operatorname{coth}\left(\theta_{1}\right)+b_{4} \cos \left(\theta_{2}\right)-\sin \left(\theta_{2}\right) \operatorname{coth}\left(\theta_{3}\right) b_{5} b_{6}\right) \cos \left(\theta_{4}\right)^{2}$

$+\left(\begin{array}{c}\left(b_{7} b_{2} \cos \left(\theta_{2}\right)+\left(b_{9} \operatorname{coth}\left(\theta_{3}\right)+\tanh \left(\theta_{3}\right) b_{8}\right) \sin \left(\theta_{2}\right)\right) \operatorname{coth}\left(\theta_{1}\right) \\ +\left(b_{11} \operatorname{coth}\left(\theta_{3}\right)+b_{10} \tanh \left(\theta_{3}\right)\right) \cos \left(\theta_{2}\right)+\sin \left(\theta_{2}\right) b_{5} b_{12}\end{array}\right) \sin \left(\theta_{4}\right) \cos \left(\theta_{4}\right)$

$+\left(\left(\operatorname{coth}\left(\theta_{3}\right) b_{13} b_{2} \cos \left(\theta_{2}\right)+b_{14} \sin \left(\theta_{2}\right)\right) \operatorname{coth}\left(\theta_{1}\right)+b_{15} \cos \left(\theta_{2}\right)+\sin \left(\theta_{2}\right) \tanh \left(\theta_{3}\right) b_{5} b_{16}\right) \sin \left(\theta_{4}\right)^{2}=0$

where coefficients $b_{r}$ depending on $t_{r, s, \sim}$ appear from the analytical calculation of the determinants. The coefficients have been calculated and are listed in Ref. [41]. The zeroes of the left hand side of Eq. (46) in fact represent the length of the shell whose $m, n$ mode resonates at the assumed frequency $\omega_{m, n}$, where the lowest zero (the smallest length) is for $m=1$ symmetric mode, the next one is for $m=3$ symmetric mode, etc. According to this notation there are $m+1$ axial nodes (the cross-sections where $w=0$ ) for a mode number $m$. In case of a non-rotating shell $(\Omega=0)$, assuming frequencies $\pm\left|\omega_{m, n}\right|$ yields the same length for either the positive or the negative frequency $\omega_{m, n}$. However for $\Omega \neq 0$ this is not the case, presented later in this paper. It is thus necessary to consider the mode shapes and natural frequencies for a number of different cases regarding the form of the roots to the polynomial in Eq. (27), not only for Rayleigh modes, but also for various other types of modes that may occur. In conclusion eight types of roots were detected that occur with both stationary and rotating shells having both ends free and they are listed in Table 2. In the following seven subsections the procedure outlined in Section 2.1 is followed for each remaining type of roots listed in Table 2 and the expressions for mode shapes and the boundary condition determinants analogue to that of Eq. (46) are developed. 
Table 2

Summary of the eight types or roots of the characteristic bi-quartic polynomial.

\begin{tabular}{ll}
\hline Case & Roots \\
\hline 1 & $\pm \alpha_{1}, \pm \mathrm{i} \gamma_{2}, \pm(p \pm \mathrm{i} q)$ \\
2 & $\pm(f \pm \mathrm{i} g), \pm(p \pm \mathrm{i} q)$ \\
3 & $\pm \alpha_{1}, \pm \alpha_{2}, \pm(p \pm \mathrm{i} q)$ \\
4 & $\pm \alpha_{1}, \pm \alpha_{2}, \pm \alpha_{3}, \pm \alpha_{4}$ \\
5 & $\pm \alpha_{1}, \pm \alpha_{2}, \pm \alpha_{3}, \pm \mathrm{i} \gamma_{4}$ \\
6 & $\pm \alpha_{1}, \pm \alpha_{2}, \pm \mathrm{i} \gamma_{3}, \pm \mathrm{i} \gamma_{4}$ \\
7 & $\pm \alpha_{1}, \pm \mathrm{i} \gamma_{2}, \pm \mathrm{i} \gamma_{3}, \pm \mathrm{i} \gamma_{4}$ \\
8 & $\pm \mathrm{i} \gamma_{1}, \pm \mathrm{i} \gamma_{2}, \pm(p \pm \mathrm{i} q)$ \\
\hline
\end{tabular}

\subsection{Case 2: eight complex roots}

A case is considered now when the roots have the form of $\pm(f \pm \mathrm{i} g), \pm(p \pm \mathrm{i} q)$. Then the three displacement components for symmetric modes are given by:

$$
\begin{aligned}
W(x)= & F_{1} \cosh \left(\frac{p x}{a}\right) \cos \left(\frac{q x}{a}\right)+F_{2} \sinh \left(\frac{p x}{a}\right) \sin \left(\frac{q x}{a}\right) \\
& +F_{3} \cosh \left(\frac{f x}{a}\right) \cos \left(\frac{g x}{a}\right)+F_{4} \sinh \left(\frac{f x}{a}\right) \sin \left(\frac{g x}{a}\right) \\
V(x)= & \left(d_{1} F_{1}+d_{2} F_{2}\right) \cosh \left(\frac{p x}{a}\right) \cos \left(\frac{q x}{a}\right) \\
& +\left(d_{1} F_{2}-d_{2} F_{1}\right) \sinh \left(\frac{p x}{a}\right) \sin \left(\frac{q x}{a}\right) \\
& +\left(d_{5} F_{3}+d_{6} F_{4}\right) \cosh \left(\frac{f x}{a}\right) \cos \left(\frac{g x}{a}\right) \\
& +\left(d_{5} F_{4}-d_{6} F_{3}\right) \sinh \left(\frac{f x}{a}\right) \sin \left(\frac{g x}{a}\right)
\end{aligned}
$$

$$
\begin{aligned}
U(x)= & \left(d_{3} F_{1}+d_{4} F_{2}\right) \sinh \left(\frac{p x}{a}\right) \cos \left(\frac{q x}{a}\right) \\
& +\left(d_{3} F_{2}-d_{4} F_{1}\right) \cosh \left(\frac{p x}{a}\right) \sin \left(\frac{q x}{a}\right) \\
& +\left(d_{7} F_{3}+d_{8} F_{4}\right) \sinh \left(\frac{f x}{a}\right) \cos \left(\frac{g x}{a}\right) \\
& +\left(d_{7} F_{4}-d_{8} F_{2}\right) \cosh \left(\frac{f x}{a}\right) \sin \left(\frac{g x}{a}\right) .
\end{aligned}
$$

The constants $d_{r}$ can again be calculated using Eqs. (31) and (32), yielding this time:

$d_{1}=\mathfrak{R}\left(V_{0} / W_{0}\right)_{r}$ with $\alpha_{r}=p+\mathrm{i} q$,

$d_{2}=\mathfrak{J}\left(V_{0} / W_{0}\right)_{r}$ with $\alpha_{r}=p+\mathrm{i} q$,

$d_{3}=\mathfrak{R}\left(U_{0} / W_{0}\right)_{r}$ with $\alpha_{r}=p+\mathrm{i} q$,

$d_{4}=\mathfrak{s}\left(U_{0} / W_{0}\right)_{r}$ with $\alpha_{r}=p+\mathrm{i} q$,

$d_{5}=\mathfrak{R}\left(V_{0} / W_{0}\right)_{r}$ with $\alpha_{r}=f+\mathrm{ig}$,

$d_{6}=\mathfrak{I}\left(V_{0} / W_{0}\right)_{r}$ with $\alpha_{r}=f+\mathrm{i} g$,

$d_{7}=\mathfrak{R}\left(U_{0} / W_{0}\right)_{r}$ with $\alpha_{r}=f+\mathrm{ig}$,

$d_{8}=\mathfrak{I}\left(U_{0} / W_{0}\right)_{r}$ with $\alpha_{r}=f+\mathrm{i} g$,

Next the same procedure as before is followed. That is, to substitute Eq. (48) into (28) for the radial displacement component, and also Eqs. (49) and (50) into analogue expressions for tangential and axial displacement components, that gives a set of displacement components $u, v$ and $w$. The displacement components are then substituted into the boundary condition expressions (Eqs. (38)-(41)), which after assuming $x=L / 2$ and putting into a matrix form gives:
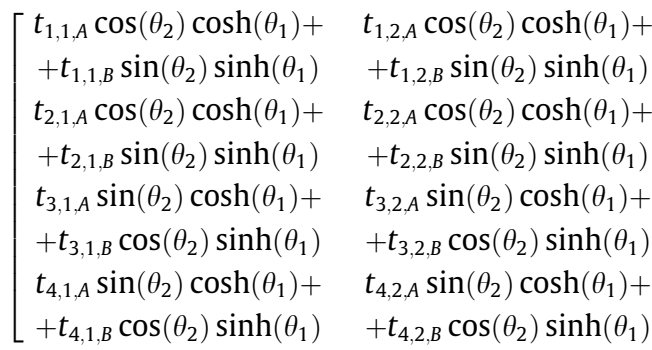

$t_{1,3, A} \cos \left(\theta_{4}\right) \cosh \left(\theta_{3}\right)+$

$+t_{1,3, B} \sin \left(\theta_{4}\right) \sinh \left(\theta_{3}\right)$

$t_{2,3, A} \cos \left(\theta_{4}\right) \cosh \left(\theta_{3}\right)+$

$+t_{2,3, B} \sin \left(\theta_{4}\right) \sinh \left(\theta_{3}\right)$

$t_{3,3, A} \sin \left(\theta_{4}\right) \cosh \left(\theta_{3}\right)+$

$+t_{3,3, B} \cos \left(\theta_{4}\right) \sinh \left(\theta_{3}\right)$

$t_{4,3, A} \sin \left(\theta_{4}\right) \cosh \left(\theta_{3}\right)+$

$+t_{4,3, B} \cos \left(\theta_{4}\right) \sinh \left(\theta_{3}\right)$

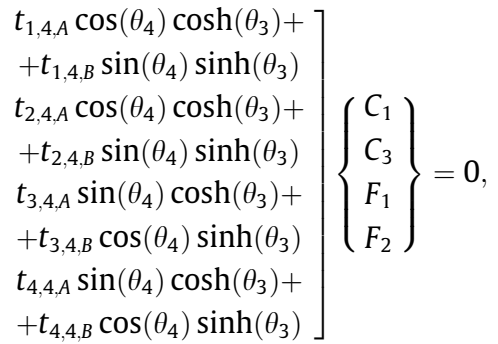

where $\theta_{1}=\frac{p L}{2 a}, \theta_{2}=\frac{q L}{2 a}, \theta_{3}=\frac{f L}{2 a}$ and $\theta_{4}=\frac{g L}{2 a}$. The coefficients $t_{r, s, \sim}$ depend on the roots $p, q, f, g$, the constants $d_{r}, n$ and $\mu$, and are listed in the Appendix to the paper. The determinant of the matrix in Eq. (52), after being divided by $\sinh \left(\theta_{1}\right) \cosh \left(\theta_{1}\right) \sinh \left(\theta_{3}\right)$ $\cosh \left(\theta_{3}\right)$ for better numerical behaviour, is given by:

$$
\begin{aligned}
& \left(\cos \left(\theta_{2}\right)\right)^{2}\left(\begin{array}{l}
\left(\cos \left(\theta_{4}\right)\right)^{2}\left(b_{3}+b_{1} \operatorname{coth}\left(\theta_{1}\right) \tanh \left(\theta_{3}\right)-b_{2} \operatorname{coth}\left(\theta_{3}\right) \tanh \left(\theta_{1}\right)\right)+ \\
+\sin \left(\theta_{4}\right) \cos \left(\theta_{4}\right)\left(b_{4} \operatorname{coth}\left(\theta_{1}\right)+b_{5} \operatorname{coth}\left(\theta_{3}\right)-b_{6} \tanh \left(\theta_{1}\right)-b_{7} \tanh \left(\theta_{3}\right)\right)+ \\
+\left(\sin \left(\theta_{4}\right)\right)^{2}\left(b_{10}+b_{8} \operatorname{coth}\left(\theta_{1}\right) \operatorname{coth}\left(\theta_{3}\right)-b_{9} \tanh \left(\theta_{1}\right) \tanh \left(\theta_{3}\right)\right)
\end{array}\right) \\
& +\cos \left(\theta_{2}\right) \sin \left(\theta_{2}\right)\left(\begin{array}{l}
\left(\cos \left(\theta_{4}\right)\right)^{2}\left(b_{11} \operatorname{coth}\left(\theta_{1}\right)-b_{12} \operatorname{coth}\left(\theta_{3}\right)+b_{13} \tanh \left(\theta_{1}\right)+b_{14} \tanh \left(\theta_{3}\right)\right) \\
+\sin \left(\theta_{4}\right) \cos \left(\theta_{4}\right)\left(\begin{array}{l}
b_{19}+\left(b_{15} \operatorname{coth}\left(\theta_{3}\right)-b_{16} \tanh \left(\theta_{3}\right)\right) \operatorname{coth}\left(\theta_{1}\right) \\
-b_{17} \tanh \left(\theta_{1}\right) \operatorname{coth}\left(\theta_{3}\right)-b_{18} \tanh \left(\theta_{3}\right) \tanh \left(\theta_{1}\right)
\end{array}\right) \\
+\left(\sin \left(\theta_{4}\right)\right)^{2}\left(b_{20} \operatorname{coth}\left(\theta_{1}\right)+b_{21} \operatorname{coth}\left(\theta_{3}\right)+b_{22} \tanh \left(\theta_{1}\right)-b_{23} \tanh \left(\theta_{3}\right)\right)
\end{array}\right), \\
& -\left(\sin \left(\theta_{2}\right)\right)^{2}\left(\begin{array}{l}
\left(\cos \left(\theta_{4}\right)\right)^{2}\left(b_{26}+b_{24} \operatorname{coth}\left(\theta_{1}\right) \operatorname{coth}\left(\theta_{3}\right)+b_{25} \tanh \left(\theta_{1}\right) \tanh \left(\theta_{3}\right)\right) \\
+\sin \left(\theta_{4}\right) \cos \left(\theta_{4}\right)\left(b_{27} \operatorname{coth}\left(\theta_{1}\right)+b_{28} \operatorname{coth}\left(\theta_{3}\right)+b_{31} \tanh \left(\theta_{1}\right)+b_{29} \tanh \left(\theta_{3}\right)\right) \\
+\left(\sin \left(\theta_{4}\right)\right)^{2}\left(b_{33}+b_{32} \operatorname{coth}\left(\theta_{3}\right) \tanh \left(\theta_{1}\right)+b_{30} \operatorname{coth}\left(\theta_{1}\right) \tanh \left(\theta_{3}\right)\right)
\end{array}\right)=0
\end{aligned}
$$


where coefficients $b_{r}$ appear from the calculation of the determinants and can be found in [41]. In case of anti-symmetric modes it must be $C_{1}=-C_{3}, C_{2}=C_{4}, C_{5}=-C_{7}$, and $C_{6}=C_{8}$ so that:

$$
\begin{aligned}
W(x) & =F_{5} \sinh \left(\frac{p x}{a}\right) \cos \left(\frac{q x}{a}\right)+F_{6} \cosh \left(\frac{p x}{a}\right) \sin \left(\frac{q x}{a}\right) \\
& +F_{7} \sinh \left(\frac{f x}{a}\right) \cos \left(\frac{g x}{a}\right)+F_{8} \cosh \left(\frac{f x}{a}\right) \sin \left(\frac{g x}{a}\right),
\end{aligned}
$$

In a similar way to the previous cases, Eq. (55) is substituted into (28) for the radial displacement component, and also Eqs. (56) and (57) into analogue expressions for tangential and axial displacement components, giving a set of displacement components $u, v$ and $w$. These can be substituted into the boundary condition expressions (Eqs. (38)-(41)), which after assuming $x=L / 2$ and putting into a matrix form gives:

$$
\left[\begin{array}{cccc}
t_{1,1} \cosh \left(\theta_{1}\right) & t_{1,2} \cosh \left(\theta_{2}\right) & t_{1,3, A} \cos \left(\theta_{4}\right) \cosh \left(\theta_{3}\right)+ & t_{1,4, A} \cos \left(\theta_{4}\right) \cosh \left(\theta_{3}\right)+ \\
& +t_{1,3, B} \sin \left(\theta_{4}\right) \sinh \left(\theta_{3}\right) & +t_{1,4, B} \sin \left(\theta_{4}\right) \sinh \left(\theta_{3}\right) \\
t_{2,1} \cosh \left(\theta_{1}\right) & t_{2,2} \cosh \left(\theta_{2}\right) & t_{2,3, A} \cos \left(\theta_{4}\right) \cosh \left(\theta_{3}\right)+ & t_{2,4, A} \cos \left(\theta_{4}\right) \cosh \left(\theta_{3}\right)+ \\
& & +t_{2,3, B} \sin \left(\theta_{4}\right) \sinh \left(\theta_{3}\right) & +t_{2,4, B} \sin \left(\theta_{4}\right) \sinh \left(\theta_{3}\right) \\
& & t_{3,3, A} \sin \left(\theta_{4}\right) \cosh \left(\theta_{3}\right)+ & t_{3,4, A} \sin \left(\theta_{4}\right) \cosh \left(\theta_{3}\right)+ \\
t_{3,1} \sinh \left(\theta_{1}\right) & t_{3,2} \sinh \left(\theta_{2}\right) & +t_{3,3, B} \cos \left(\theta_{4}\right) \sinh \left(\theta_{3}\right) & +t_{3,4, B} \cos \left(\theta_{4}\right) \sinh \left(\theta_{3}\right) \\
& & t_{4,3, A} \sin \left(\theta_{4}\right) \cosh \left(\theta_{3}\right)+ & t_{4,4, A} \sin \left(\theta_{4}\right) \cosh \left(\theta_{3}\right)+ \\
t_{4,1} \sinh \left(\theta_{1}\right) & t_{4,2} \sinh \left(\theta_{2}\right) & +t_{4,3, B} \cos \left(\theta_{4}\right) \sinh \left(\theta_{3}\right) & +t_{4,4, B} \cos \left(\theta_{4}\right) \sinh \left(\theta_{3}\right)
\end{array}\right]\left\{\begin{array}{l}
C_{1} \\
C_{3} \\
F_{1} \\
F_{2}
\end{array}\right\}=0,
$$

with similar expressions for $V(x)$ and $U(x)$. After recalculating the determinant for the anti-symmetric modes an expression analogue to Eq. (53) results, which can be obtained by substituting $\operatorname{coth}\left(\theta_{1}\right) \leftrightarrow \tanh \left(\theta_{1}\right)$ and $\tanh \left(\theta_{3}\right) \leftrightarrow \operatorname{coth}\left(\theta_{3}\right)$, into Eq. (53). where $\theta_{1}=\frac{\alpha_{1} L}{2 a}, \theta_{2}=\frac{\alpha_{2} L}{2 a}, \theta_{3}=\frac{p L}{2 a}$ and $\theta_{4}=\frac{q L}{2 a}$.

The coefficients $t_{r, s, \sim}$ depend on the roots $\alpha_{1}, \alpha_{2}, p, q$, the constants $d_{r}, n$ and $\mu$, and are listed in the Appendix to the paper. The determinant of the matrix in Eq. (59), is now divided by $\sinh \left(\theta_{1}\right) \sinh \left(\theta_{2}\right) \sinh \left(\theta_{3}\right) \cosh \left(\theta_{3}\right)$ for better numerical behaviour, yielding:

$$
\begin{aligned}
& \left(\cos \left(\theta_{4}\right)\right)^{2}\left(b_{4}+\left(b_{1} \tanh \left(\theta_{2}\right)+b_{2} \tanh \left(\theta_{3}\right)\right) \operatorname{coth}\left(\theta_{1}\right)-b_{3} \operatorname{coth}\left(\theta_{3}\right) \tanh \left(\theta_{2}\right)\right) \\
& +\sin \left(\theta_{4}\right) \cos \left(\theta_{4}\right)\left(\begin{array}{l}
\left(\left(b_{5} \operatorname{coth}\left(\theta_{3}\right)+b_{6} \tanh \left(\theta_{3}\right)\right) \tanh \left(\theta_{2}\right)+b_{7}\right) \operatorname{coth}\left(\theta_{1}\right) \\
+b_{8} \tanh \left(\theta_{2}\right)+b_{9} \operatorname{coth}\left(\theta_{3}\right)+b_{10} \tanh \left(\theta_{3}\right)
\end{array}\right) \\
& +\left(\sin \left(\theta_{4}\right)\right)^{2}\left(b_{14}+\left(b_{11} \tanh \left(\theta_{2}\right)+b_{13} \operatorname{coth}\left(\theta_{3}\right)\right) \operatorname{coth}\left(\theta_{1}\right)+b_{12} \tanh \left(\theta_{2}\right) \tanh \left(\theta_{3}\right)\right)=0
\end{aligned}
$$

\subsection{Case 3: four real and four complex roots}

The next case is with the roots having the form of $\pm \alpha_{1}, \pm \alpha_{2}, \pm(p \pm \mathrm{i} q)$, which three displacement components for symmetric modes are given by:

$$
\begin{aligned}
W(x)= & C_{1} \cosh \left(\frac{\alpha_{1} x}{a}\right)+C_{3} \cosh \left(\frac{\alpha_{2} x}{a}\right)+F_{1} \cosh \left(\frac{p x}{a}\right) \cos \left(\frac{q x}{a}\right) \\
& +F_{2} \sinh \left(\frac{p x}{a}\right) \sin \left(\frac{q x}{a}\right), \\
V(x)= & d_{1} C_{1} \cosh \left(\frac{\alpha_{1} x}{a}\right)+d_{3} C_{3} \cosh \left(\frac{\alpha_{2} x}{a}\right)+\left(d_{5} F_{1}+d_{6} F_{2}\right) \\
& \times \cosh \left(\frac{p x}{a}\right) \cos \left(\frac{q x}{a}\right)+\left(d_{5} F_{2}-d_{6} F_{1}\right) \sinh \left(\frac{p x}{a}\right) \sin \left(\frac{q x}{a}\right), \\
U(x)= & d_{2} C_{1} \sinh \left(\frac{\alpha_{1} x}{a}\right)+d_{4} C_{3} \sinh \left(\frac{\alpha_{2} x}{a}\right)+\left(d_{7} F_{1}+d_{8} F_{2}\right) \sinh \left(\frac{p x}{a}\right) \\
\times & \cos \left(\frac{q x}{a}\right)+\left(d_{7} F_{2}-d_{8} F_{1}\right) \cosh \left(\frac{p x}{a}\right) \sin \left(\frac{q x}{a}\right) .
\end{aligned}
$$

Also the Eqs. (31) and (32) are used to calculate the constants $d_{r}$, obtaining:

$$
\begin{aligned}
& d_{1}=\left(V_{0} / W_{0}\right)_{r} \text { with } \alpha_{r}=\alpha_{1}, \\
& d_{2}=\left(U_{0} / W_{0}\right)_{r} \text { with } \alpha_{r}=\alpha_{1}, \\
& d_{3}=\left(V_{0} / W_{0}\right)_{r} \text { with } \alpha_{r}=\alpha_{2}, \\
& d_{4}=\left(U_{0} / W_{0}\right)_{r} \text { with } \alpha_{r}=\alpha_{2}, \\
& d_{5}=\mathfrak{R}\left(V_{0} / W_{0}\right)_{r} \text { with } \alpha_{r}=p+\mathrm{i} q, \\
& d_{6}=\mathfrak{I}\left(V_{0} / W_{0}\right)_{r} \text { with } \alpha_{r}=p+\mathrm{i} q, \\
& d_{7}=\mathfrak{R}\left(U_{0} / W_{0}\right)_{r} \text { with } \alpha_{r}=p+\mathrm{i} q, \\
& d_{8}=\mathfrak{I}\left(U_{0} / W_{0}\right)_{r} \text { with } \alpha_{r}=p+\mathrm{i} q .
\end{aligned}
$$

where coefficients $b_{r}$ are given in [41].

In case of anti-symmetric modes it must be $C_{1}=C_{3}=0$, $C_{5}=-C_{7}$, and $C_{6}=C_{8}$ so that:

$$
\begin{aligned}
W(x)= & C_{2} \sinh \left(\frac{\alpha_{1} x}{a}\right)+C_{4} \sinh \left(\frac{\alpha_{2} x}{a}\right)+F_{3} \\
& \times \sinh \left(\frac{p x}{a}\right) \cos \left(\frac{q x}{a}\right)+F_{4} \cosh \left(\frac{p x}{a}\right) \sin \left(\frac{q x}{a}\right),
\end{aligned}
$$

with similar expressions for $V(x)$ and $U(x)$. After recalculating the determinant for the anti-symmetric modes an expression analogue to Eq. (60) results, which can be obtained by substituting $\operatorname{coth}\left(\theta_{1}\right) \leftrightarrow \tanh \left(\theta_{1}\right), \quad \tanh \left(\theta_{2}\right) \leftrightarrow \operatorname{coth}\left(\theta_{2}\right), \quad$ and $\quad \tanh \left(\theta_{3}\right) \leftrightarrow$ $\operatorname{coth}\left(\theta_{3}\right)$ into Eq. (60).

\subsection{Case 4: eight real roots}

The following case considered is with the roots in the form $\pm \alpha_{1}, \pm \alpha_{2}, \pm \alpha_{3}, \pm \alpha_{4}$. In case of symmetric modes:

$$
\begin{aligned}
W(x)= & C_{1} \cosh \left(\frac{x \alpha_{1}}{a}\right)+C_{2} \cosh \left(\frac{x \alpha_{2}}{a}\right)+C_{3} \cosh \left(\frac{x \alpha_{3}}{a}\right) \\
& +C_{4} \cosh \left(\frac{x \alpha_{4}}{a}\right), \\
V(x)= & d_{1} C_{1} \cosh \left(\frac{x \alpha_{1}}{a}\right)+d_{3} C_{2} \cosh \left(\frac{x \alpha_{2}}{a}\right) \\
& +d_{5} C_{3} \cosh \left(\frac{x \alpha_{3}}{a}\right)+d_{7} C_{4} \cosh \left(\frac{x \alpha_{4}}{a}\right), \\
U(x)= & d_{2} C_{1} \sinh \left(\frac{x \alpha_{1}}{a}\right)+d_{4} C_{2} \sinh \left(\frac{x \alpha_{2}}{a}\right)+d_{6} C_{3} \sinh \left(\frac{x \alpha_{3}}{a}\right) \\
& +d_{8} C_{4} \sinh \left(\frac{x \alpha_{4}}{a}\right) .
\end{aligned}
$$


The constants $d_{r}$ can again be calculated using Eqs. (31) and (32) as:

$d_{1}=\left(V_{0} / W_{0}\right)_{r}$ with $\alpha_{r}=\alpha_{1}$,

$d_{2}=\left(U_{0} / W_{0}\right)_{r}$ with $\alpha_{r}=\alpha_{1}$,

$d_{3}=\left(V_{0} / W_{0}\right)_{r}$ with $\alpha_{r}=\alpha_{2}$,

$d_{4}=\left(U_{0} / W_{0}\right)_{r}$ with $\alpha_{r}=\alpha_{2}$,

$d_{5}=\left(V_{0} / W_{0}\right)_{r}$ with $\alpha_{r}=\alpha_{3}$,

$d_{6}=\left(U_{0} / W_{0}\right)_{r}$ with $\alpha_{r}=\alpha_{3}$,

$d_{7}=\left(V_{0} / W_{0}\right)_{r}$ with $\alpha_{r}=\alpha_{4}$,

$d_{8}=\left(U_{0} / W_{0}\right)_{r}$ with $\alpha_{r}=\alpha_{4}$.

The boundary conditions are satisfied by requiring that:

$\left[\begin{array}{llll}t_{1,1} \cosh \left(\theta_{1}\right) & t_{1,2} \cosh \left(\theta_{2}\right) & t_{1,3} \cosh \left(\theta_{3}\right) & t_{1,4} \cosh \left(\theta_{4}\right) \\ t_{2,1} \cosh \left(\theta_{1}\right) & t_{2,2} \cosh \left(\theta_{2}\right) & t_{2,3} \cosh \left(\theta_{3}\right) & t_{2,4} \cosh \left(\theta_{4}\right) \\ t_{3,1} \sinh \left(\theta_{1}\right) & t_{3,2} \sinh \left(\theta_{2}\right) & t_{3,3} \sinh \left(\theta_{3}\right) & t_{3,4} \sinh \left(\theta_{4}\right) \\ t_{4,1} \sinh \left(\theta_{1}\right) & t_{4,2} \sinh \left(\theta_{2}\right) & t_{4,3} \sinh \left(\theta_{3}\right) & t_{4,4} \sinh \left(\theta_{4}\right)\end{array}\right]\left\{\begin{array}{l}C_{1} \\ C_{2} \\ C_{3} \\ C_{4}\end{array}\right\}=0$

After being divided by $\sinh \left(\theta_{1}\right) \cosh \left(\theta_{2}\right) \sinh \left(\theta_{3}\right) \cosh \left(\theta_{4}\right)$, the determinant obtained is:

$\left(-b_{1} \operatorname{coth}\left(\theta_{3}\right) \tanh \left(\theta_{2}\right) \tanh \left(\theta_{4}\right)+b_{3} \tanh \left(\theta_{2}\right)+b_{2} \tanh \left(\theta_{4}\right)\right) \operatorname{coth}\left(\theta_{1}\right)$ $+\left(-b_{5} \tanh \left(\theta_{2}\right)-b_{4} \tanh \left(\theta_{4}\right)\right) \operatorname{coth}\left(\theta_{3}\right)+b_{6}=0$

where $\theta_{1}=\frac{\alpha_{1} L}{2 a}, \theta_{2}=\frac{\alpha_{2} L}{2 a}, \theta_{3}=\frac{\alpha_{3} L}{2 a}$ and $\theta_{4}=\frac{\alpha_{4} L}{2 a}$.

In case of anti-symmetric modes it must be $C_{1}=C_{2}=C_{3}=$ $C_{4}=0$, so that:

$$
\begin{aligned}
W(x)= & C_{5} \sinh \left(\frac{x \alpha_{1}}{a}\right)+C_{6} \sinh \left(\frac{x \alpha_{2}}{a}\right)+C_{7} \sinh \left(\frac{x \alpha_{3}}{a}\right) \\
& +C_{8} \sinh \left(\frac{x \alpha_{4}}{a}\right),
\end{aligned}
$$

with similar expressions for $V(x)$ and $U(x)$. After recalculating the determinant for the anti-symmetric modes an expression analogue to Eq. (67) results, which can be obtained by substituting $\operatorname{coth}\left(\theta_{1}\right) \leftrightarrow \tanh \left(\theta_{1}\right), \quad \tanh \left(\theta_{2}\right) \leftrightarrow \operatorname{coth}\left(\theta_{2}\right), \quad \operatorname{coth}\left(\theta_{3}\right) \leftrightarrow \tanh \left(\theta_{3}\right)$, $\tanh \left(\theta_{4}\right) \leftrightarrow \operatorname{coth}\left(\theta_{4}\right)$, into Eq. (67).

\subsection{Case 5: six real and two imaginary roots}

The form of the roots $\pm \alpha_{1}, \pm \alpha_{2}, \pm \alpha_{3}, \pm \mathbf{i} \gamma_{4}$ is the next case considered. In case of symmetric modes the displacement components are given by:

$W(x)=C_{1} \cosh \left(\frac{x \alpha_{1}}{a}\right)+C_{2} \cosh \left(\frac{x \alpha_{2}}{a}\right)+C_{3} \cosh \left(\frac{x \alpha_{3}}{a}\right)+C_{4} \cos \left(\frac{x \gamma_{4}}{a}\right)$,

$V(x)=d_{1} C_{1} \cosh \left(\frac{x \alpha_{1}}{a}\right)+d_{3} C_{2} \cosh \left(\frac{x \alpha_{2}}{a}\right)+d_{5} C_{3} \cosh \left(\frac{x \alpha_{3}}{a}\right)$ $+d_{7} C_{4} \cos \left(\frac{x \gamma_{4}}{a}\right)$

$U(x)=d_{2} C_{1} \sinh \left(\frac{x \alpha_{1}}{a}\right)+d_{4} C_{2} \sinh \left(\frac{x \alpha_{2}}{a}\right)+d_{6} C_{3} \sinh \left(\frac{x \alpha_{3}}{a}\right)$

$$
-d_{8} C_{4} \sin \left(\frac{x \gamma_{4}}{a}\right) \text {. }
$$

The constants $d_{r}$ can be calculated using Eqs. (31) and (32) as:

$d_{1}=\left(V_{0} / W_{0}\right)_{r}$ with $\alpha_{r}=\alpha_{1}$,

$d_{2}=\left(U_{0} / W_{0}\right)_{r}$ with $\alpha_{r}=\alpha_{1}$

$d_{3}=\left(V_{0} / W_{0}\right)_{r}$ with $\alpha_{r}=\alpha_{2}$,

$d_{4}=\left(U_{0} / W_{0}\right)_{r}$ with $\alpha_{r}=\alpha_{2}$

$d_{5}=\left(V_{0} / W_{0}\right)_{r}$ with $\alpha_{r}=\alpha_{3}$,

$d_{6}=\left(U_{0} / W_{0}\right)_{r}$ with $\alpha_{r}=\alpha_{3}$,

$d_{7}=\mathfrak{R}\left(V_{0} / W_{0}\right)_{r}$ with $\alpha_{r}=\mathrm{i} \gamma_{4}$,

$d_{8}=\mathfrak{J}\left(U_{0} / W_{0}\right)_{r}$ with $\alpha_{r}=\mathrm{i} \gamma_{4}$.
The boundary conditions are satisfied by requiring that:

$$
\left[\begin{array}{llll}
t_{1,1} \cosh \left(\theta_{1}\right) & t_{1,2} \cosh \left(\theta_{2}\right) & t_{1,3} \cosh \left(\theta_{3}\right) & t_{1,4} \cos \left(\theta_{4}\right) \\
t_{2,1} \cosh \left(\theta_{1}\right) & t_{2,2} \cosh \left(\theta_{2}\right) & t_{2,3} \cosh \left(\theta_{3}\right) & t_{2,4} \cos \left(\theta_{4}\right) \\
t_{3,1} \sinh \left(\theta_{1}\right) & t_{3,2} \sinh \left(\theta_{2}\right) & t_{3,3} \sinh \left(\theta_{3}\right) & t_{3,4} \sin \left(\theta_{4}\right) \\
t_{4,1} \sinh \left(\theta_{1}\right) & t_{4,2} \sinh \left(\theta_{2}\right) & t_{4,3} \sinh \left(\theta_{3}\right) & t_{4,4} \sin \left(\theta_{4}\right)
\end{array}\right]\left\{\begin{array}{l}
C_{1} \\
C_{2} \\
C_{3} \\
C_{4}
\end{array}\right\}=0,
$$

so that the following expression results from dividing the determinant by $\sinh \left(\theta_{3}\right) \sinh \left(\theta_{1}\right) \cosh \left(\theta_{2}\right)$ and equating to zero:

$\left(-b_{1} \operatorname{coth}\left(\theta_{3}\right) \sin \left(\theta_{4}\right) \tanh \left(\theta_{2}\right)+b_{2} \cos \left(\theta_{4}\right) \tanh \left(\theta_{2}\right)+b_{3} \sin \left(\theta_{4}\right)\right) \operatorname{coth}\left(\theta_{1}\right)$ $+\left(-b_{4} \cos \left(\theta_{4}\right) \tanh \left(\theta_{2}\right)-b_{5} \sin \left(\theta_{4}\right)\right) \operatorname{coth}\left(\theta_{3}\right)+b_{6} \cos \left(\theta_{4}\right)=0$

where $\theta_{1}=\frac{\alpha_{1} L}{2 a}, \theta_{2}=\frac{\alpha_{2} L}{2 a}, \theta_{3}=\frac{\alpha_{3} L}{2 a}$ and $\theta_{4}=\frac{\gamma_{4} L}{2 a}$.

In case of anti-symmetric modes it must be $C_{1}=C_{2}=C_{3}=$ $C_{4}=0$, so that:

$$
\begin{aligned}
W(x)= & C_{5} \sinh \left(\frac{x \alpha_{1}}{a}\right)+C_{6} \sinh \left(\frac{x \alpha_{2}}{a}\right)+C_{7} \sinh \left(\frac{x \alpha_{3}}{a}\right) \\
& +C_{8} \sin \left(\frac{x \gamma_{4}}{a}\right),
\end{aligned}
$$

with similar expressions for $V(x)$ and $U(x)$. After recalculating the determinant for the anti-symmetric modes an expression analogue to Eq. (74) results, which can be obtained by substituting $\operatorname{coth}\left(\theta_{1}\right) \leftrightarrow \tanh \left(\theta_{1}\right), \tanh \left(\theta_{2}\right) \leftrightarrow \operatorname{coth}\left(\theta_{2}\right), \quad \operatorname{coth}\left(\theta_{3}\right) \leftrightarrow \tanh \left(\theta_{3}\right)$, $\sin \left(\theta_{4}\right) \rightarrow-\cos \left(\theta_{4}\right), \cos \left(\theta_{4}\right) \rightarrow \sin \left(\theta_{4}\right)$ into Eq. (74).

\subsection{Case 6: four real and four imaginary roots}

The case 6 is for the roots that take the form $\pm \alpha_{1}, \pm \alpha_{2}, \pm \mathbf{i} \gamma_{3}, \pm \mathbf{i} \gamma_{4}$. In such a case the three displacement components are given by:

$$
\begin{aligned}
W(x)= & C_{1} \cosh \left(\frac{x \alpha_{1}}{a}\right)+C_{2} \cosh \left(\frac{x \alpha_{2}}{a}\right)+C_{3} \cos \left(\frac{x \gamma_{3}}{a}\right) \\
& +C_{4} \cos \left(\frac{x \gamma_{4}}{a}\right), \\
V(x)= & d_{1} C_{1} \cosh \left(\frac{x \alpha_{1}}{a}\right)+d_{3} C_{2} \cosh \left(\frac{x \alpha_{2}}{a}\right)+d_{5} C_{3} \cos \left(\frac{x \gamma_{3}}{a}\right) \\
& +d_{7} C_{4} \cos \left(\frac{x \gamma_{4}}{a}\right), \\
U(x)= & d_{2} C_{1} \sinh \left(\frac{x \alpha_{1}}{a}\right)+d_{4} C_{2} \sinh \left(\frac{x \alpha_{2}}{a}\right)-d_{6} C_{3} \sin \left(\frac{x \gamma_{3}}{a}\right) \\
& -d_{8} C_{4} \sin \left(\frac{x \gamma_{4}}{a}\right) .
\end{aligned}
$$

The constants $d_{r}$ can again be calculated using Eqs. (31) and (32) as: $d_{1}=\left(V_{0} / W_{0}\right)_{r}$ with $\alpha_{r}=\alpha_{1}$,

$d_{2}=\left(U_{0} / W_{0}\right)_{r}$ with $\alpha_{r}=\alpha_{1}$,

$d_{3}=\left(V_{0} / W_{0}\right)_{r}$ with $\alpha_{r}=\alpha_{2}$,

$d_{4}=\left(U_{0} / W_{0}\right)_{r}$ with $\alpha_{r}=\alpha_{2}$,

$d_{5}=\mathfrak{R}\left(V_{0} / W_{0}\right)_{r}$ with $\alpha_{r}=\mathrm{i} \gamma_{3}$,

$d_{6}=\mathfrak{J}\left(U_{0} / W_{0}\right)_{r}$ with $\alpha_{r}=\mathrm{i} \gamma_{3}$,

$d_{7}=\mathfrak{R}\left(V_{0} / W_{0}\right)_{r}$ with $\alpha_{r}=\mathrm{i} \gamma_{4}$,

$d_{8}=\mathfrak{J}\left(U_{0} / W_{0}\right)_{r}$ with $\alpha_{r}=\mathrm{i} \gamma_{4}$.

The boundary conditions are satisfied if:

$$
\left[\begin{array}{llll}
t_{1,1} \cosh \left(\theta_{1}\right) & t_{1,2} \cosh \left(\theta_{2}\right) & t_{1,3} \cos \left(\theta_{3}\right) & t_{1,4} \cos \left(\theta_{4}\right) \\
t_{2,1} \cosh \left(\theta_{1}\right) & t_{2,2} \cosh \left(\theta_{2}\right) & t_{2,3} \cos \left(\theta_{3}\right) & t_{2,4} \cos \left(\theta_{4}\right) \\
t_{3,1} \sinh \left(\theta_{1}\right) & t_{3,2} \sinh \left(\theta_{2}\right) & t_{3,3} \sin \left(\theta_{3}\right) & t_{3,4} \sin \left(\theta_{4}\right) \\
t_{4,1} \sinh \left(\theta_{1}\right) & t_{4,2} \sinh \left(\theta_{2}\right) & t_{4,3} \sin \left(\theta_{3}\right) & t_{4,4} \sin \left(\theta_{4}\right)
\end{array}\right]\left\{\begin{array}{l}
C_{1} \\
C_{2} \\
C_{3} \\
C_{4}
\end{array}\right\}=0,
$$


or after normalising the determinant by $\sinh \left(\theta_{2}\right) \sinh \left(\theta_{1}\right)$ :

$\left(b_{1} \operatorname{coth}\left(\theta_{2}\right) \sin \left(\theta_{3}\right) \sin \left(\theta_{4}\right)-b_{2} \cos \left(\theta_{3}\right) \sin \left(\theta_{4}\right)+b_{3} \cos \left(\theta_{4}\right) \sin \left(\theta_{3}\right)\right) \operatorname{coth}\left(\theta_{1}\right)$ $+\left(-b_{4} \cos \left(\theta_{3}\right) \sin \left(\theta_{4}\right)+b_{5} \cos \left(\theta_{4}\right) \sin \left(\theta_{3}\right)\right) \operatorname{coth}\left(\theta_{2}\right)-b_{6} \cos \left(\theta_{3}\right) \cos \left(\theta_{4}\right)=0$ '

where $\theta_{1}=\frac{\alpha_{1} L}{2 a}, \theta_{2}=\frac{\alpha_{2} L}{2 a}, \theta_{3}=\frac{\gamma_{3} L}{2 a}$ and $\theta_{4}=\frac{\gamma_{4} L}{2 a}$.

In case of anti-symmetric modes it must be $C_{1}=C_{2}=C_{3}=$ $C_{4}=0$, so that:

$$
\begin{aligned}
W(x)= & C_{5} \sinh \left(\frac{x \alpha_{1}}{a}\right)+C_{6} \sinh \left(\frac{x \alpha_{2}}{a}\right)+C_{7} \sin \left(\frac{x \gamma_{3}}{a}\right) \\
& +C_{8} \sin \left(\frac{x \gamma_{4}}{a}\right),
\end{aligned}
$$

with similar expressions for $V(x)$ and $U(x)$. After recalculating the determinant for the anti-symmetric modes an expression analogue to Eq. (81) results, which can be obtained by substituting $\operatorname{coth}\left(\theta_{1}\right) \leftrightarrow \tanh \left(\theta_{1}\right), \quad \operatorname{coth}\left(\theta_{2}\right) \leftrightarrow \tanh \left(\theta_{2}\right), \quad \sin \left(\theta_{3}\right) \rightarrow-\cos \left(\theta_{3}\right)$, $\cos \left(\theta_{3}\right) \rightarrow \sin \left(\theta_{3}\right), \sin \left(\theta_{4}\right) \rightarrow-\cos \left(\theta_{4}\right), \cos \left(\theta_{4}\right) \rightarrow \sin \left(\theta_{4}\right)$ into Eq. (81).

\subsection{Case 7: two real and six imaginary roots}

In case of $\pm \alpha_{1}, \pm \mathbf{i} \gamma_{2}, \pm \mathbf{i} \gamma_{3}, \pm \mathbf{i} \gamma_{4}$ the three displacement components are:

$W(x)=C_{1} \cosh \left(\frac{x \alpha_{1}}{a}\right)+C_{2} \cos \left(\frac{x \gamma_{2}}{a}\right)+C_{3} \cos \left(\frac{x \gamma_{3}}{a}\right)+C_{4} \cos \left(\frac{x \gamma_{4}}{a}\right)$,

$$
\begin{aligned}
V(x)= & d_{1} C_{1} \cosh \left(\frac{x \alpha_{1}}{a}\right)+d_{3} C_{2} \cos \left(\frac{x \gamma_{2}}{a}\right)+d_{5} C_{3} \cos \left(\frac{x \gamma_{3}}{a}\right) \\
& +d_{7} C_{4} \cos \left(\frac{x \gamma_{4}}{a}\right), \\
U(x)= & d_{2} C_{1} \sinh \left(\frac{x \alpha_{1}}{a}\right)-d_{4} C_{2} \sin \left(\frac{x \gamma_{2}}{a}\right)-d_{6} C_{3} \sin \left(\frac{x \gamma_{3}}{a}\right) \\
& -d_{8} C_{4} \sin \left(\frac{x \gamma_{4}}{a}\right) .
\end{aligned}
$$

The constants $d_{r}$ can be calculated using Eqs. (31) and (32) as:

$d_{1}=\left(V_{0} / W_{0}\right)_{r}$ with $\alpha_{r}=\alpha_{1}$,

$d_{2}=\left(U_{0} / W_{0}\right)_{r}$ with $\alpha_{r}=\alpha_{1}$,

$d_{3}=\mathfrak{R}\left(V_{0} / W_{0}\right)_{r}$ with $\alpha_{r}=\mathrm{i} \gamma_{2}$,

$d_{4}=\mathfrak{I}\left(U_{0} / W_{0}\right)_{r}$ with $\alpha_{r}=\mathrm{i} \gamma_{2}$,

$d_{5}=\mathfrak{R}\left(V_{0} / W_{0}\right)_{r}$ with $\alpha_{r}=\mathrm{i} \gamma_{3}$,

$d_{6}=\mathfrak{s}\left(U_{0} / W_{0}\right)_{r}$ with $\alpha_{r}=\mathrm{i} \gamma_{3}$,

$d_{7}=\mathfrak{R}\left(V_{0} / W_{0}\right)_{r}$ with $\alpha_{r}=\mathrm{i} \gamma_{4}$,

$d_{8}=\mathfrak{J}\left(U_{0} / W_{0}\right)_{r}$ with $\alpha_{r}=\mathrm{i} \gamma_{4}$.

The boundary conditions are satisfied if:

$$
\left[\begin{array}{llll}
t_{1,1} \cosh \left(\theta_{1}\right) & t_{1,2} \cos \left(\theta_{2}\right) & t_{1,3} \cos \left(\theta_{3}\right) & t_{1,4} \cos \left(\theta_{4}\right) \\
t_{2,1} \cosh \left(\theta_{1}\right) & t_{2,2} \cos \left(\theta_{2}\right) & t_{2,3} \cos \left(\theta_{3}\right) & t_{2,4} \cos \left(\theta_{4}\right) \\
t_{3,1} \sinh \left(\theta_{1}\right) & t_{3,2} \sin \left(\theta_{2}\right) & t_{3,3} \sin \left(\theta_{3}\right) & t_{3,4} \sin \left(\theta_{4}\right) \\
t_{4,1} \sinh \left(\theta_{1}\right) & t_{4,2} \sin \left(\theta_{2}\right) & t_{4,3} \sin \left(\theta_{3}\right) & t_{4,4} \sin \left(\theta_{4}\right)
\end{array}\right]\left\{\begin{array}{l}
C_{1} \\
C_{2} \\
C_{3} \\
C_{4}
\end{array}\right\}=0,
$$

or after normalising the determinant of the $4 \times 4$ matrix by $\sinh \left(\theta_{1}\right)$ :

$\left(b_{1} \cos \left(\theta_{2}\right) \sin \left(\theta_{3}\right) \sin \left(\theta_{4}\right)+\left(-b_{2} \cos \left(\theta_{3}\right) \sin \left(\theta_{4}\right)+b_{3} \cos \left(\theta_{4}\right) \sin \left(\theta_{3}\right)\right)\right.$ $\left.\times \sin \left(\theta_{2}\right)\right) \operatorname{coth}\left(\theta_{1}\right)+\left(-b_{4} \cos \left(\theta_{3}\right) \sin \left(\theta_{4}\right)+b_{5} \cos \left(\theta_{4}\right) \sin \left(\theta_{3}\right)\right)$

$$
\times \cos \left(\theta_{2}\right)-b_{6} \cos \left(\theta_{3}\right) \cos \left(\theta_{4}\right) \sin \left(\theta_{2}\right)=0,
$$

where $\theta_{1}=\frac{\alpha_{1} L}{2 a}, \theta_{2}=\frac{\gamma_{2} L}{2 a}, \theta_{3}=\frac{\gamma_{3} L}{2 a}$ and $\theta_{4}=\frac{\gamma_{4} L}{2 a}$. In case of antisymmetric modes it must be $C_{1}=C_{2}=C_{3}=C_{4}=0$, so that:

$W(x)=C_{5} \sinh \left(\frac{x \alpha_{1}}{a}\right)+C_{6} \sin \left(\frac{x \gamma_{2}}{a}\right)+C_{7} \sin \left(\frac{x \gamma_{3}}{a}\right)+C_{8} \sin \left(\frac{x \gamma_{4}}{a}\right)$,

with similar expressions for $V(x)$ and $U(x)$. After recalculating the determinant for the anti-symmetric modes an expression analogue to Eq. (88) results, which can be obtained by substituting $\operatorname{coth}\left(\theta_{1}\right) \leftrightarrow \tanh \left(\theta_{1}\right), \quad \cos \left(\theta_{2}\right) \rightarrow \sin \left(\theta_{2}\right), \quad \sin \left(\theta_{2}\right) \rightarrow-\cos \left(\theta_{2}\right)$, $\cos \left(\theta_{3}\right) \rightarrow \sin \left(\theta_{3}\right), \quad \sin \left(\theta_{3}\right) \rightarrow-\cos \left(\theta_{3}\right), \quad \cos \left(\theta_{4}\right) \rightarrow \sin \left(\theta_{4}\right)$, $\sin \left(\theta_{4}\right) \rightarrow-\cos \left(\theta_{4}\right)$ into Eq. (88).

\subsection{Case 8: four imaginary and four complex roots}

The final case considered is when the roots are $\pm \mathbf{i} \gamma_{1}, \pm \mathbf{i} \gamma_{2}$, $\pm(p \pm \mathrm{i} q)$.

$$
\begin{aligned}
W(x)= & C_{1} \cos \left(\frac{x \gamma_{1}}{a}\right)+C_{3} \cos \left(\frac{x \gamma_{2}}{a}\right)+F_{1} \cosh \left(\frac{x p}{a}\right) \cos \left(\frac{x q}{a}\right) \\
& +F_{2} \sinh \left(\frac{x p}{a}\right) \sin \left(\frac{x q}{a}\right) \\
V(x)= & d_{1} C_{1} \cos \left(\frac{x \gamma_{1}}{a}\right)+d_{3} C_{3} \cos \left(\frac{x \gamma_{2}}{a}\right) \\
& +\left(d_{5} F_{1}+d_{6} F_{2}\right) \cosh \left(\frac{x p}{a}\right) \cos \left(\frac{x q}{a}\right) \\
& +\left(d_{5} F_{2}-d_{6} F_{1}\right) \sinh \left(\frac{x p}{a}\right) \sin \left(\frac{x q}{a}\right) \\
U(x)= & -d_{2} C_{1} \sin \left(\frac{x \gamma_{1}}{a}\right)-d_{4} C_{3} \sin \left(\frac{x \gamma_{2}}{a}\right) \\
& +\left(d_{7} F_{1}+d_{8} F_{2}\right) \sinh \left(\frac{x p}{a}\right) \cos \left(\frac{x q}{a}\right) \\
& +\left(d_{7} F_{2}-d_{8} F_{1}\right) \cosh \left(\frac{x p}{a}\right) \sin \left(\frac{x q}{a}\right) .
\end{aligned}
$$

The constants $d_{r}$ can be calculated using Eqs. (31) and (32) as:

$$
\begin{aligned}
& d_{1}=\mathfrak{R}\left(V_{0} / W_{0}\right)_{r} \text { with } \alpha_{r}=\mathrm{i} \gamma_{1}, \\
& d_{2}=\mathfrak{I}\left(U_{0} / W_{0}\right)_{r} \text { with } \alpha_{r}=\mathrm{i} \gamma_{1}, \\
& d_{3}=\mathfrak{R}\left(V_{0} / W_{0}\right)_{r} \text { with } \alpha_{r}=\mathrm{i} \gamma_{2}, \\
& d_{4}=\mathfrak{I}\left(U_{0} / W_{0}\right)_{r} \text { with } \alpha_{r}=\mathrm{i} \gamma_{2}, \\
& d_{5}=\mathfrak{R}\left(V_{0} / W_{0}\right)_{r} \text { with } \alpha_{r}=p+\mathrm{i} q, \\
& d_{6}=\mathfrak{I}\left(U_{0} / W_{0}\right)_{r} \text { with } \alpha_{r}=p+\mathrm{i} q, \\
& d_{7}=\mathfrak{R}\left(V_{0} / W_{0}\right)_{r} \text { with } \alpha_{r}=p+\mathrm{i} q, \\
& d_{8}=\mathfrak{I}\left(U_{0} / W_{0}\right)_{r} \text { with } \alpha_{r}=p+\mathrm{i} q .
\end{aligned}
$$

The boundary conditions are satisfied if:

$\left[\begin{array}{cccc}t_{1,1} \cos \left(\theta_{1}\right) & t_{1,2} \cos \left(\theta_{2}\right) & t_{1,3, A} \cos \left(\theta_{4}\right) \cosh \left(\theta_{3}\right)+ & t_{1,4, A} \cos \left(\theta_{4}\right) \cosh \left(\theta_{3}\right)+ \\ & & +t_{1,3, B} \sin \left(\theta_{4}\right) \sinh \left(\theta_{3}\right) & +t_{1,4, B} \sin \left(\theta_{4}\right) \sinh \left(\theta_{3}\right) \\ t_{2,1} \cos \left(\theta_{1}\right) & t_{2,2} \cos \left(\theta_{2}\right) & t_{2,3, A} \cos \left(\theta_{4}\right) \cosh \left(\theta_{3}\right)+ & t_{2,4, A} \cos \left(\theta_{4}\right) \cosh \left(\theta_{3}\right)+ \\ & & +t_{2,3, B} \sin \left(\theta_{4}\right) \sinh \left(\theta_{3}\right) & +t_{2,4, B} \sin \left(\theta_{4}\right) \sinh \left(\theta_{3}\right) \\ & & t_{3,3, A} \sin \left(\theta_{4}\right) \cosh \left(\theta_{3}\right)+ & t_{3,4, A} \sin \left(\theta_{4}\right) \cosh \left(\theta_{3}\right)+ \\ t_{3,1} \sin \left(\theta_{1}\right) & t_{3,2} \sin \left(\theta_{2}\right) & +t_{3,3, B} \cos \left(\theta_{4}\right) \sinh \left(\theta_{3}\right) & +t_{3,4, B} \cos \left(\theta_{4}\right) \sinh \left(\theta_{3}\right) \\ & & t_{4,3, A} \sin \left(\theta_{4}\right) \cosh \left(\theta_{3}\right)+ & t_{4,4, A} \sin \left(\theta_{4}\right) \cosh \left(\theta_{3}\right)+ \\ t_{4,1} \sin \left(\theta_{1}\right) & t_{4,2} \sin \left(\theta_{2}\right) & +t_{4,3, B} \cos \left(\theta_{4}\right) \sinh \left(\theta_{3}\right) & +t_{4,4, B} \cos \left(\theta_{4}\right) \sinh \left(\theta_{3}\right)\end{array}\right]\left\{\begin{array}{l}C_{1} \\ C_{3} \\ F_{1} \\ F_{2}\end{array}\right\}=0$


After dividing the determinant of the matrix in Eq. (94) by $\sinh \left(\theta_{3}\right) \sinh \left(\theta_{1}\right) \cosh \left(\theta_{2}\right) \cosh \left(\theta_{3}\right)$ the following expression is obtained which must vanish in order to have the boundary conditions satisfied:

$$
\begin{aligned}
& \left(\begin{array}{l}
\left(b_{2} \cos \left(\theta_{2}\right) \tanh \left(\theta_{3}\right)+\sin \left(\theta_{2}\right) b_{1}\right) \cos \left(\theta_{1}\right)- \\
\left(b_{4} \cos \left(\theta_{2}\right)+b_{3} \operatorname{coth}\left(\theta_{3}\right) \sin \left(\theta_{2}\right)\right) \sin \left(\theta_{1}\right)
\end{array}\right)\left(\cos \left(\theta_{4}\right)\right)^{2} \\
& +\left(\begin{array}{c}
\left(b_{7} \cos \left(\theta_{2}\right)+\sin \left(\theta_{2}\right)\left(b_{5} \operatorname{coth}\left(\theta_{3}\right)+\tanh \left(\theta_{3}\right) b_{6}\right)\right) \cos \left(\theta_{1}\right)+ \\
\left(\left(b_{9} \operatorname{coth}\left(\theta_{3}\right)+\tanh \left(\theta_{3}\right) b_{10}\right) \cos \left(\theta_{2}\right)+b_{8} \sin \left(\theta_{2}\right)\right) \sin \left(\theta_{1}\right)
\end{array}\right) \sin \left(\theta_{4}\right) \cos \left(\theta_{4}\right), \\
& +\left(\begin{array}{c}
\left(b_{13} \cos \left(\theta_{2}\right) \operatorname{coth}\left(\theta_{3}\right)+\sin \left(\theta_{2}\right) b_{11}\right) \cos \left(\theta_{1}\right)+ \\
\left(b_{14} \cos \left(\theta_{2}\right)+b_{12} \sin \left(\theta_{2}\right) \tanh \left(\theta_{3}\right)\right) \sin \left(\theta_{1}\right)
\end{array}\right)\left(\sin \left(\theta_{4}\right)\right)^{2}=0
\end{aligned}
$$

where $\theta_{1}=\frac{\gamma_{1} L}{2 a}, \theta_{2}=\frac{\gamma_{2} L}{2 a}, \theta_{3}=\frac{p L}{2 a}$ and $\theta_{4}=\frac{q L}{2 a}$.

In case of anti-symmetric modes it must be $C_{1}=C_{3}=0$, $C_{5}=-C_{7}$, and $C_{6}=C_{8}$ so that:

$$
\begin{aligned}
W(x)= & C_{2} \sin \left(\frac{x \gamma_{1}}{a}\right)+C_{4} \sin \left(\frac{x \gamma_{2}}{a}\right)+F_{3} \sinh \left(\frac{x p}{a}\right) \cos \left(\frac{x q}{a}\right) \\
& +F_{4} \cosh \left(\frac{x p}{a}\right) \sin \left(\frac{x q}{a}\right)
\end{aligned}
$$

with similar expressions for $V(x)$ and $U(x)$. After recalculating the determinant for the anti-symmetric modes an expression analogue to Eq. (95) results, which can be obtained by substituting $\cos \left(\theta_{1}\right) \rightarrow$ $\sin \left(\theta_{1}\right), \sin \left(\theta_{1}\right) \rightarrow-\cos \left(\theta_{1}\right), \cos \left(\theta_{2}\right) \rightarrow \sin \left(\theta_{2}\right), \sin \left(\theta_{2}\right) \rightarrow-\cos \left(\theta_{2}\right)$, $\tanh \left(\theta_{3}\right) \leftrightarrow \operatorname{coth}\left(\theta_{3}\right)$ into Eq. (95).

\section{Results}

In this section an example shell is considered and a number of mode shapes and natural frequencies are calculated. The material and geometrical properties of the example cylindrical shell are listed in Table 3.

The method for calculation of the natural frequencies is as follows. The frequency range of interest $(0-3 \mathrm{kHz})$ was divided into 30000 frequencies with an equidistant spacing of $0.1 \mathrm{~Hz}$. Then a "scan" was performed through all frequencies in the range and a change of the sign of any of the sixteen determinants (i.e. Eq (74) or Eq. (81)) was detected between two adjacent frequencies assuming the shell length as in Table 3 . The change of the sign in fact indicates the existence of a mode with its natural frequency between the two adjacent frequencies. Thus the accuracy of the calculated natural frequencies is $0.1 \mathrm{~Hz}$ or better. Fig. 3 shows the natural frequencies calculated using the methodology described above for the example shell as a function of the circumferential mode number up to $1 \mathrm{kHz}$ (plot (a)) and from $1 \mathrm{kHz}$ to $3 \mathrm{kHz}$ (plot (b)). As shown by the black continuous lines, it is in principle possible to calculate fictive natural frequencies for circumferential mode numbers which are not integers. This has no physical meaning within the scope of the present study; however it enables to visually identify the so called "branches". Each branch contains natural frequencies of modes having a constant axial mode number $m$. For example, the two branches with lowest natural frequencies per a circumferential mode number contain the Rayleigh type $(m=-1)$ and Love type modes $(m=0)$. This nomenclature is after [18].

As can be seen in Fig. 3 these two groups of modes are characterised by very similar natural frequencies if the circumferential mode number $n$ is the same, since the two branches nearly overlap. A depiction of a Love-type mode shape can be seen in Figs. 4(a), 6 (b), and 7(b). In fact, Figs. 4-12 contain two mode shapes governed by the same root type, the order of figures being the same as the order of the root types listed in Table 2. Beneath each 3D mode shape a 2D axial profile $W(x)$ is plotted that indicates $m+1$ axial nodal cross-sections.

Love modes have one axial nodal cross-section, resembling thus the rocking mode of a free-free beam, as shown by the axial profile $W(x)$ in Fig. 4(a), for example. The Rayleigh modes, on the other hand, have no axial nodal cross-sections, as shown in Fig. 5 (a) and (b), and their axial profile is similar to the even mode of a free-free beam. However, the axial profile is not completely flat; instead it tends to be curved towards the two free ends.

This curvature appears to depend on the circumferential mode number and it is slight for low values of $n$, as indicated in Fig. 5, however it increases as the circumferential mode number $n$ increases. This can be clearly seen in Figs. 6(a) and 7(a), which

\begin{tabular}{|c|c|c|c|c|c|c|c|c|c|}
\hline$a(\mathrm{~m})$ & $h(\mathrm{~m})$ & $L(\mathrm{~m})$ & $v(-)$ & $\rho\left(\mathrm{kg} \mathrm{m}^{-3}\right)$ & $E(\mathrm{GPa})$ & $k_{z}\left(\mathrm{~N} \mathrm{~m}^{-3}\right)$ & $k_{\phi}\left(\mathrm{N} \mathrm{m}^{-3}\right)$ & $p_{0}(\mathrm{~Pa})$ & $N_{x, i}\left(\mathrm{~N} \mathrm{~m}^{-1}\right)$ \\
\hline 0.1 & 0.002 & 0.2 & 0.45 & 1452 & 0.45 & 0 & 0 & 0 & 0 \\
\hline
\end{tabular}
depict a couple of Rayleigh modes with higher circumferential mode numbers of $n=16$ and $n=23$. Similarly, the axial profiles

Table 3

The geometrical and material properties of the example shell.
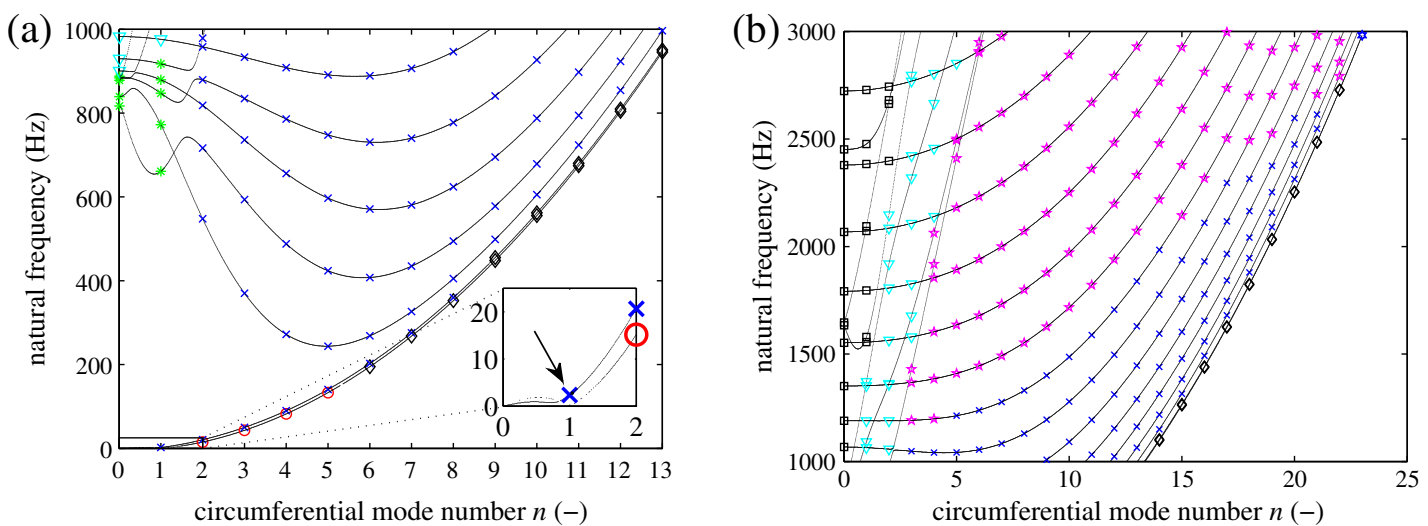

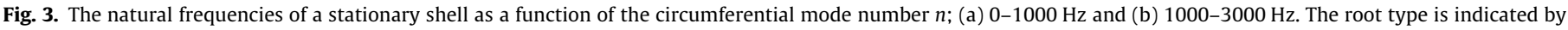

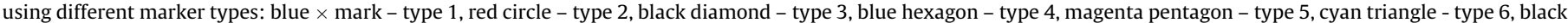

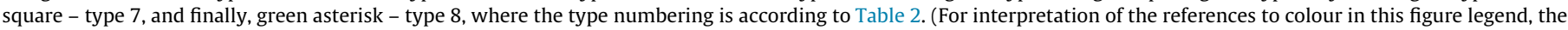
reader is referred to the web version of this article.) 

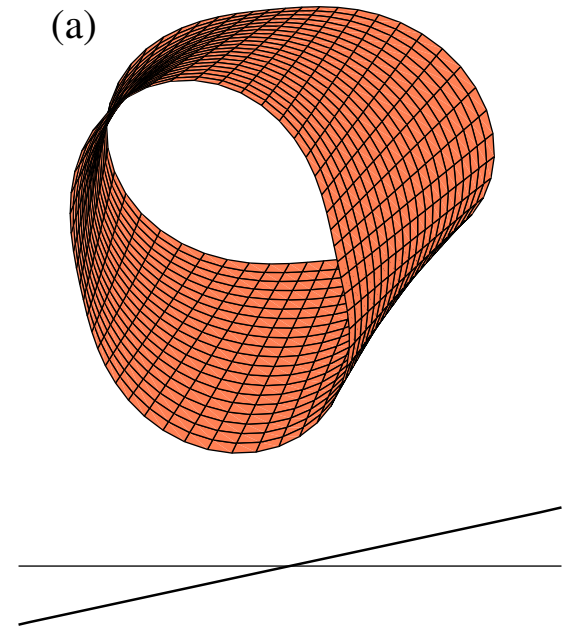

(bending, $m=0, n=2, \mathrm{f}=20.7 \mathrm{~Hz}$ )
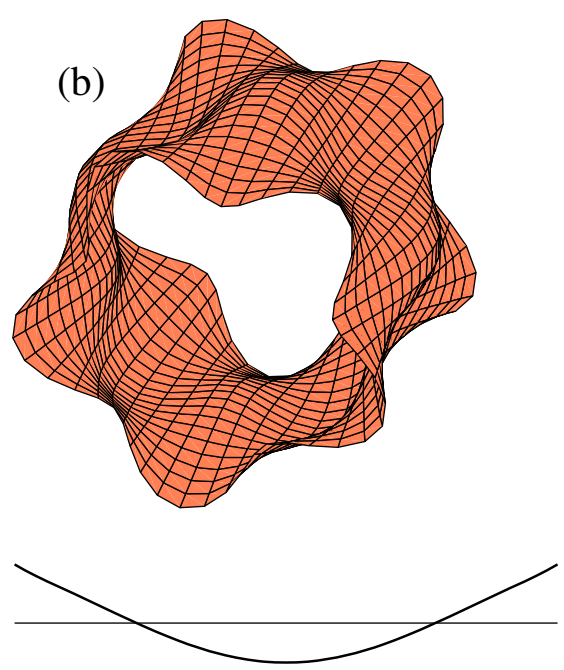

(bending, $m=1, n=5, \mathrm{f}=243.7 \mathrm{~Hz}$ )

Fig. 4. Two real, two imaginary, four complex.

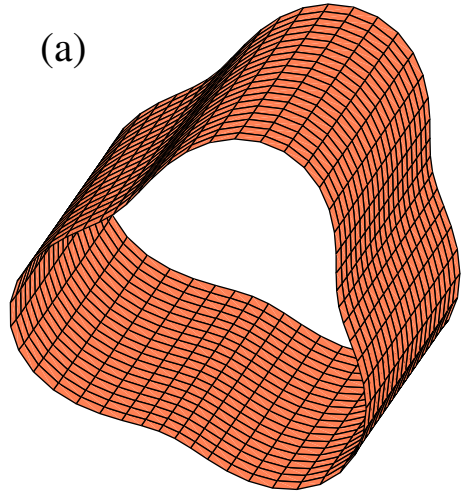

(bending, $m=-1, n=3, \mathrm{f}=42.9 \mathrm{~Hz}$ )

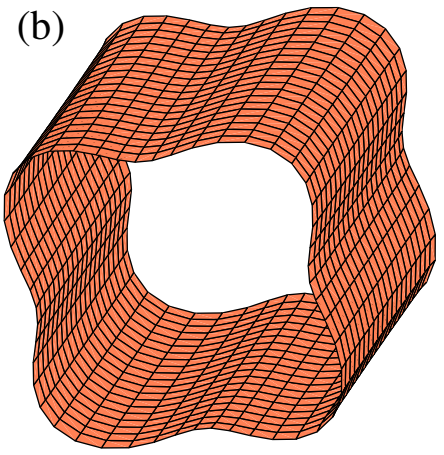

(bending, $m=-1, n=4, \mathrm{f}=82.4 \mathrm{~Hz}$ )

Fig. 5. All complex.

of Love modes also cease to resemble the rocking mode of a freefree beam due to the increased curvatures towards the ends as the circumferential mode number increases, as shown in Figs. 6 (b) and 7(b) with $n=9$ and $n=23$, respectively.

Referring now again to the natural frequencies of a stationary shell shown in Fig. 3, it is interesting to look at how the root type varies in the $(n, f)$ plane. This is made easier by highlighting the natural frequencies (at integer circumferential numbers $n$ ) using markers of different types. The markers of different types correspond to different types of the roots (Table 2) which govern different mode shapes. For which marker type corresponds to which root type see the caption to Fig. 3. It can be seen that indeed a large group of mode shapes are determined by the roots of type 1 (two real, two imaginary, four complex) as shown by the blue $\times$ symbols. It can also be seen that the Rayleigh modes are never governed by that root type. Instead, they appear to be determined by the roots of type 2, 3 and 4 . Also the modes with low circumferential mode numbers (those with $n=0$ and $n=1$ ) are governed by roots of type 6,7 and 8 . Then also a large group of "flexible" modes are characterised by the roots of type 5 ("six real two imaginary"), as shown in Fig. 3(b) by the magenta pentagon markers. The term "flexible" is conditionally used here to indicate all modes whose axial profile is not similar to the rigid body modes of a free-free beam (i.e. all modes but the Rayleigh and Love modes). The root type 1 can describe some of the Love modes (Fig. 3(a) blue $\times$ symbols). However, Rayleigh modes with no axial nodes require the root type 2 ("all complex"), Fig. 5, at low circumferential mode numbers, the root type 3 ("four real four complex"), Fig. 6(a), at intermediate circumferential mode numbers, or the root type 4 ("all real"), Fig. 7(a), at higher circumferential mode numbers.

It can be seen in Fig. 3(b) that additional branches occur, characterised by a steeper slope with respect to the horizontal axis, which in fact cross the branches containing the natural frequencies of bending modes. These branches encompass additional natural frequencies that correspond either to the longitudinal mode shapes having a pronounced axial "in plane" deformations or the shear modes having a pronounced circumferential "in plane" deforma- 


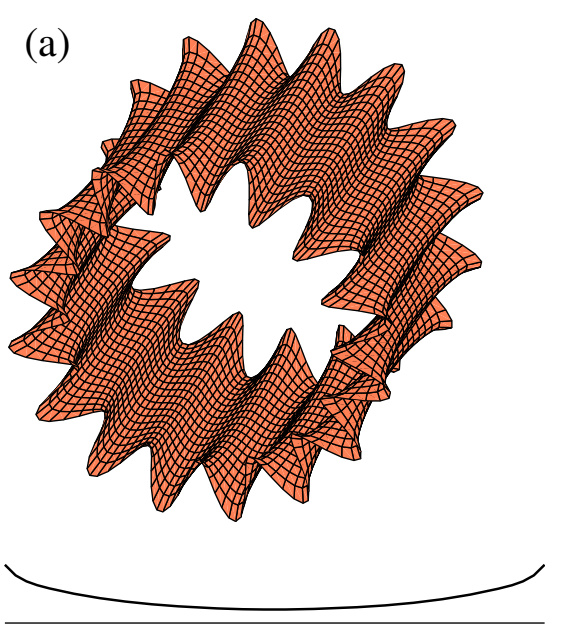

(bending, $m=-1, n=16, \mathrm{f}=1437.5 \mathrm{~Hz}$ )

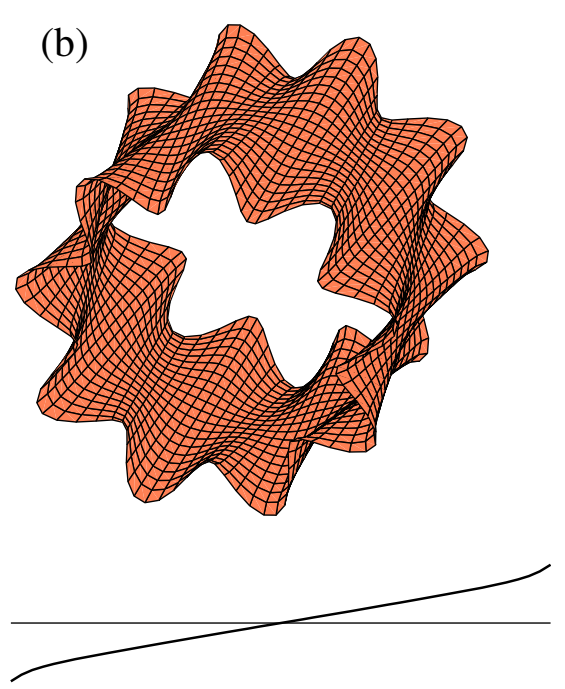

(bending, $m=0, n=9, \mathrm{f}=455.1 \mathrm{~Hz}$ )

Fig. 6. Four real, four complex.
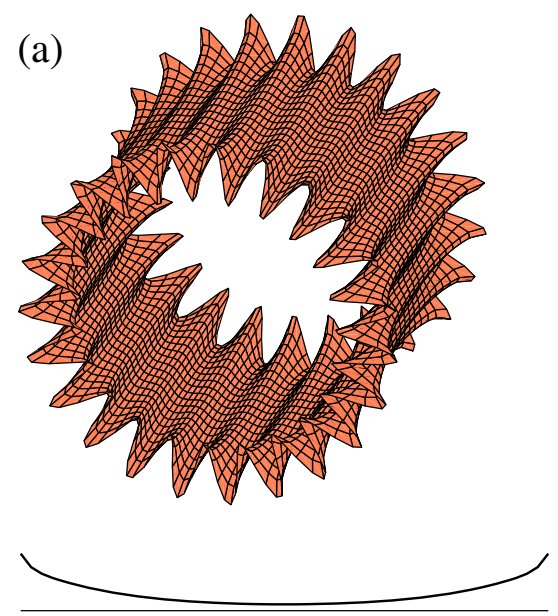

(bending, $m=-1, n=23, \mathrm{f}=2981.6 \mathrm{~Hz}$ )
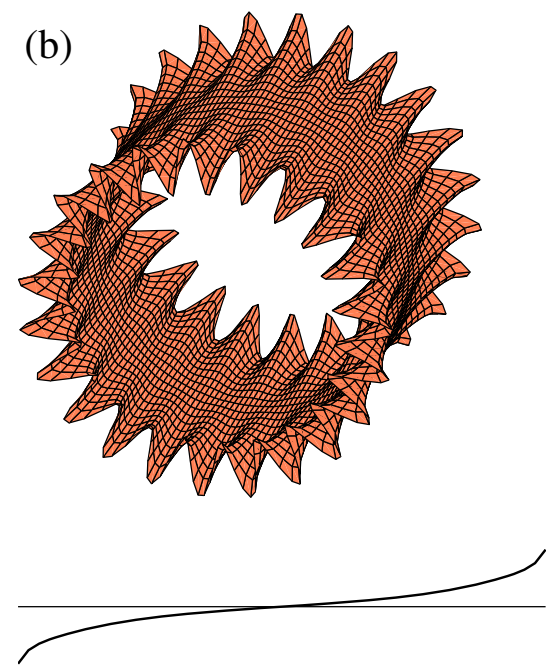

(bending, $m=0, n=23, \mathrm{f}=2983.1 \mathrm{~Hz}$ )

Fig. 7. All real.

tions of the shell. Some longitudinal mode shapes are shown in Figs. 8(b) and 10(b), whereas some shear modes are shown in Figs. 11(b) and 12(a).

It should be noted that with $m=0, n=1$, a natural frequency of about $2.3 \mathrm{~Hz}$ is calculated, as shown in Fig. 3(a), zoomed area of the plot. This is not a physically acceptable result. As can be seen in Fig. 9(a), the corresponding mode shape resembles a rigid body rocking mode of the shell which should yield a zero eigenvalue. The reason for the non-zero natural frequency lies in the assumed mode shape functions (12-14) which are unable to exactly represent rigid body rocking of the shell. The coupled radial, axial and circumferential displacement components, although roughly approximating the rocking motion, as shown in Fig. 9(a), must induce some strain (and thus potential energy) in the shell. As a result, a small natural frequency is erroneously calculated for this type of mode. This frequency is shown in Fig. 9(b) as a function of the shell length/radius for three different thickness/radius ratios. Here the length and thickness have been varied but other properties (i.e. radius) are as those indicated in Table 3. The bogus natural frequency is normalised with respect to the natural frequency of $m=-1,2$ Rayleigh mode as this is normally the lowest true natural frequency of a shell having free-free boundary conditions.

It is clear in Fig. 9(b) that the error vanishes as the length/radius increases, but it can reach $20 \%$ in case or relatively short shells with length to radius ratio near to or below unity. The relative shell thickness seems not to influence the bogus natural frequency of the rocking rigid body mode. More discussion on the rigid body modes and how they are influenced by the elastic foundation stiffness is given in Section 4 of the paper.

The results of the present exact method are now compared with the results of two experimental studies found in the literature. The first study [42] presents the results of vibration tests for aluminium-alloy cylindrical shells, with different combinations of end conditions and stiffeners. In the second study [43] experimental results of vibrational characteristics of stainless-steel cylindrical shells are given.

Three different experimental cases have been compared with the exact solution in the current work. The experimental case 1 , 

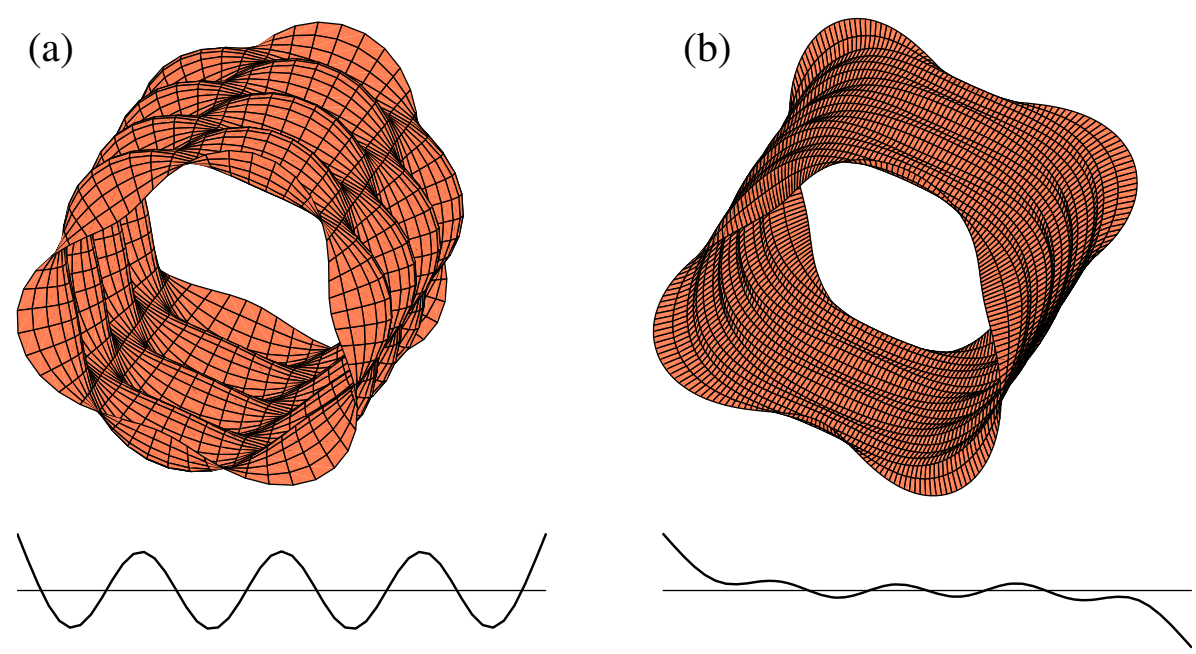

(bending, $m=7, n=3, \mathrm{f}=1191.6 \mathrm{~Hz}$ )

(longitudinal, $m=4, n=3, \mathrm{f}=1429.7 \mathrm{~Hz}$ )

Fig. 8. Six real two imaginary.
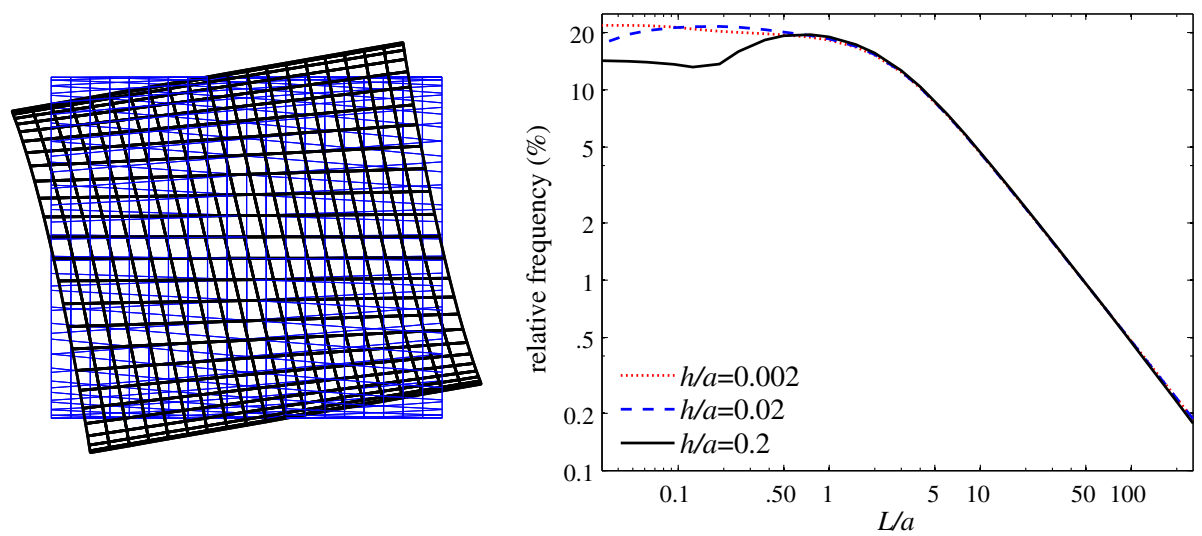

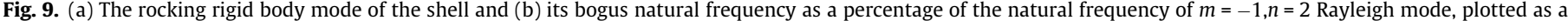
length/radius ratio.

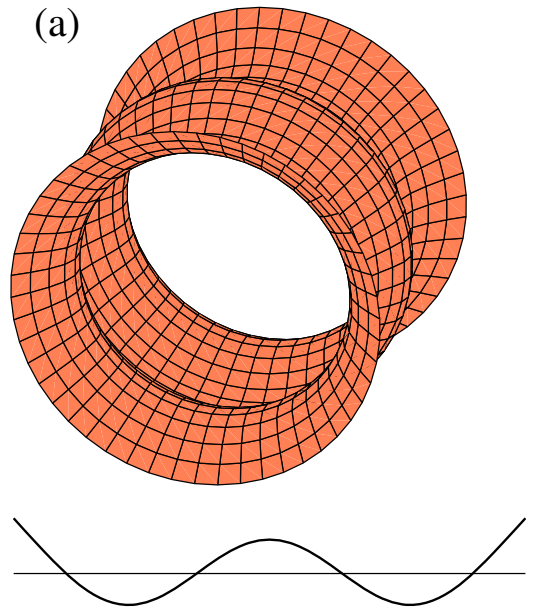

(bending, $m=3, n=0, \mathrm{f}=900.4 \mathrm{~Hz}$ )

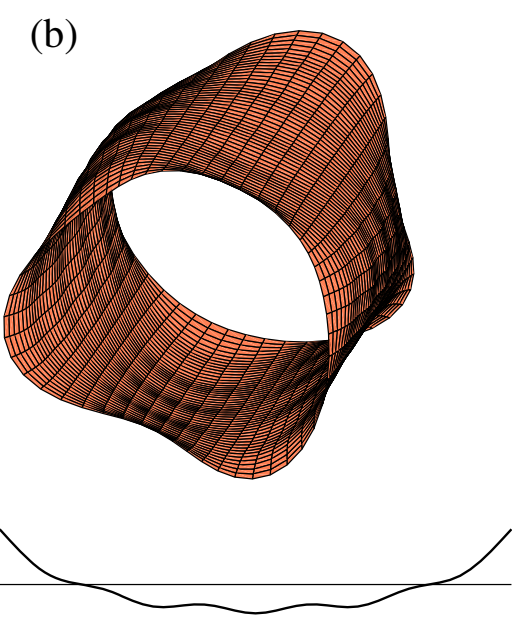

(longitudinal, $m=1, n=3, \mathrm{f}=1677.0 \mathrm{~Hz}$ )

Fig. 10. Four real four imaginary. 

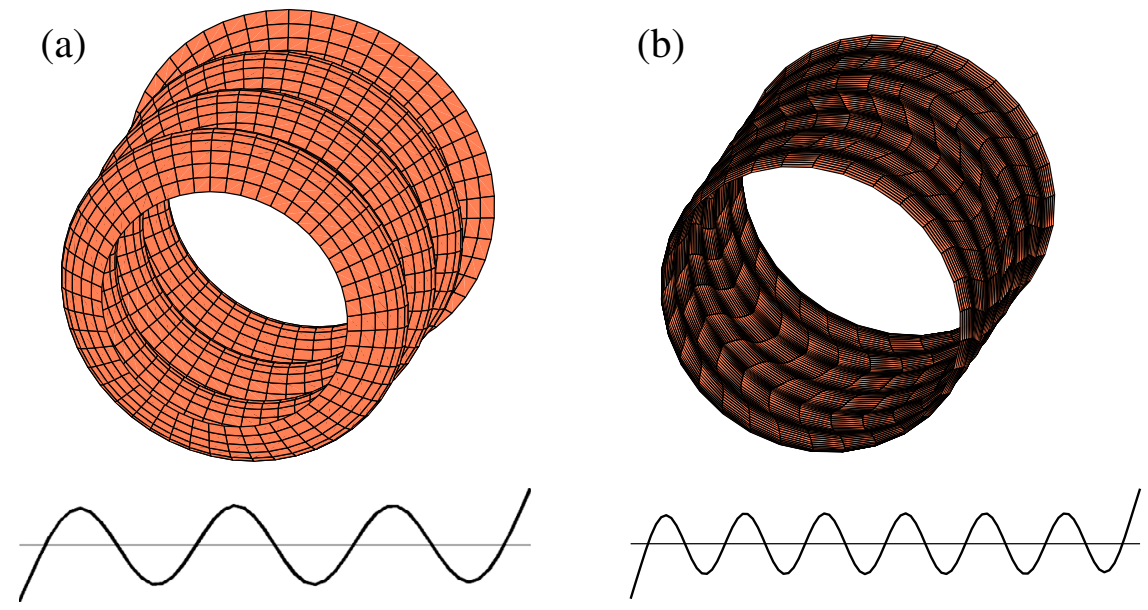

(bending, $m=6, n=0, \mathrm{f}=1067.6 \mathrm{~Hz}$ )

(shear, $m=12, n=0, \mathrm{f}=2451.8 \mathrm{~Hz}$ )

Fig. 11. Six imaginary two real.
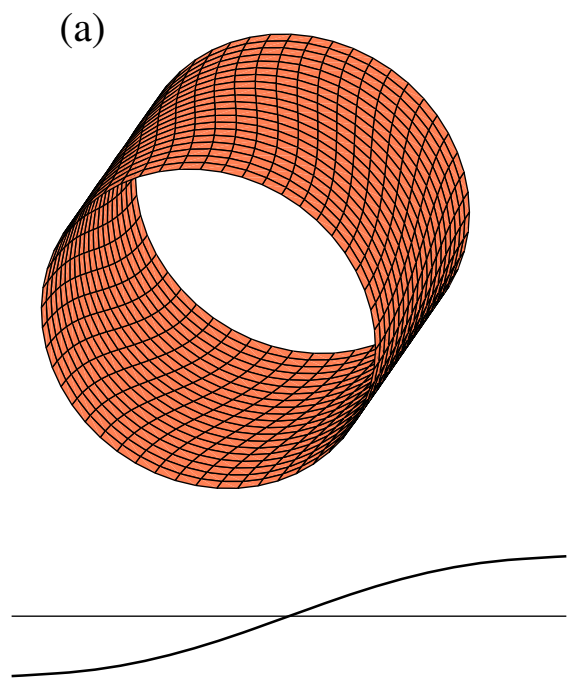

(shear, $m=0, n=0, \mathrm{f}=817.3 \mathrm{~Hz}$ )
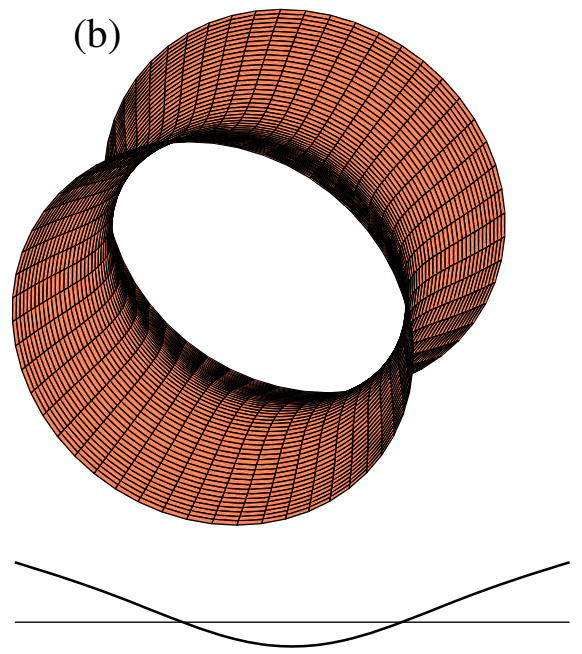

(bending, $m=1, n=0, \mathrm{f}=883.9 \mathrm{~Hz}$ )

Fig. 12. Four imaginary four complex.

Table 4

The geometrical and material properties for the comparative study.

\begin{tabular}{|c|c|c|c|c|c|c|}
\hline & $a(\mathrm{~m})$ & $h(\mathrm{~mm})$ & $L(\mathrm{~m})$ & $v(-)$ & $\rho\left(\mathrm{kg} \mathrm{m}^{-3}\right)$ & $E(\mathrm{GPa})$ \\
\hline Experimental case 1 (after [42]) & 0.242 & 0.648 & 0.638 & 0.315 & 2715 & 68.95 \\
\hline Experimental case 2 (after [43]) & 0.356 & 0.178 & 1.067 & 0.310 & 7900 & 193 \\
\hline Experimental case 3 (after [43]) & 0.254 & 0.178 & 0.457 & 0.310 & 7900 & 193 \\
\hline
\end{tabular}

after Ref. [42], is an unstiffened cylindrical aluminium-alloy shell with free-free end conditions. The experimental modal analysis was carried out using two different types of excitation shakers: an electrodynamic shaker and an air shaker. Results with both shaker types have been used for the present comparison. In the experimental cases 2 and 3, after Ref. [43], thin-walled cylindrical stainless steel shells, with free-free end conditions, and two different radius/length combinations are considered. In the experimental case 2 the results were also obtained for two different shaker types. In the experimental case 3, the natural frequencies were measured for shells with different numbers of longitudinal welding seams. The geometrical and material properties for each case are collected in Table 4.

Fig. 13 shows the natural frequencies using the same layout as in Fig. 3, where the continuous lines are the solutions using the present exact method, and the discrete symbols represent the natural frequencies taken from $[42,43]$. It can be seen that in the three cases the experimental results fall close to the analytical continuous lines indicating a very good agreement between the experimental and the analytical method. Thus the present method can be considered to agree very well with the experimental results. 

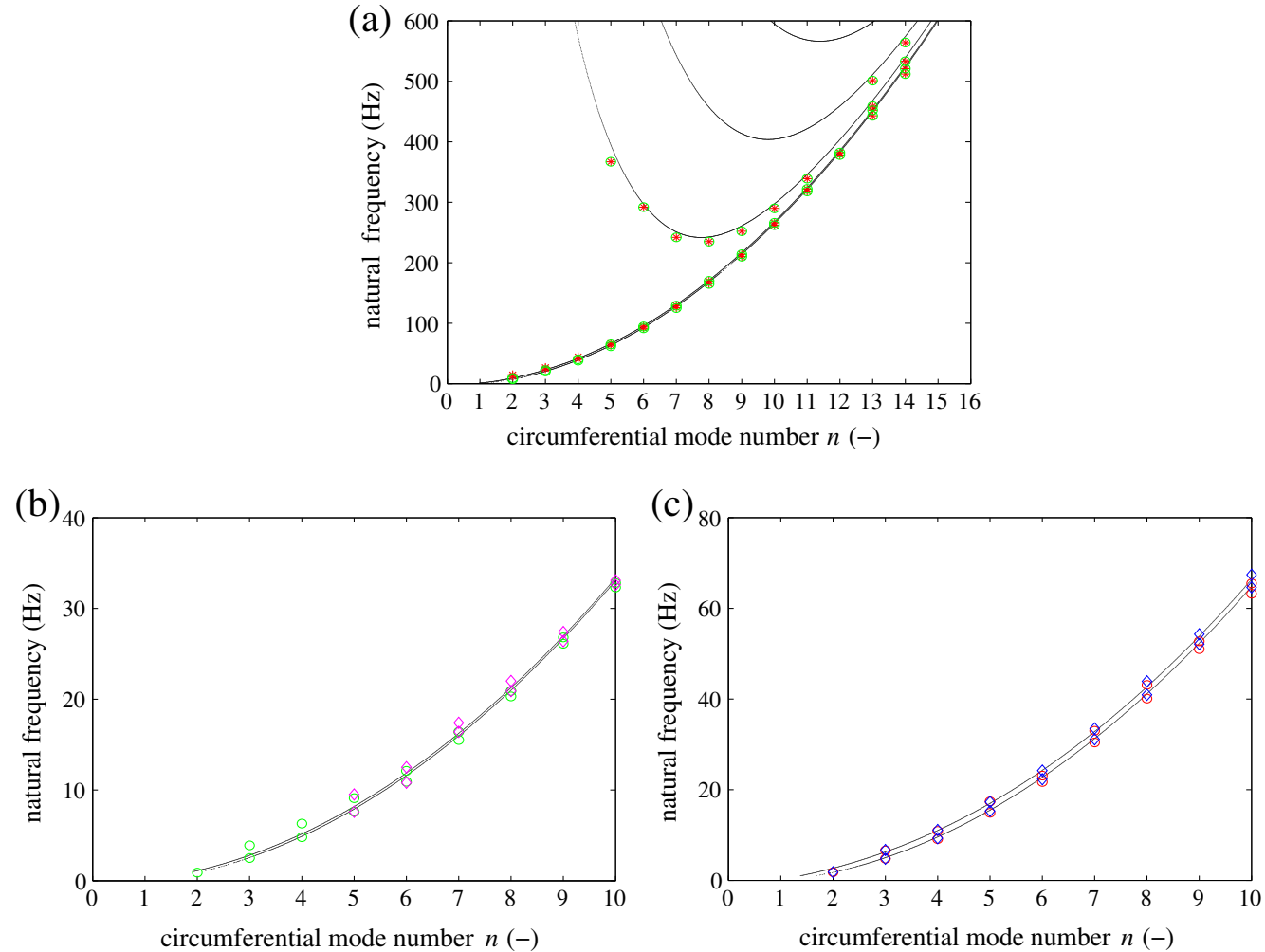

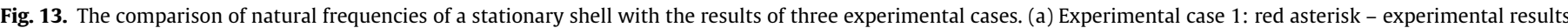

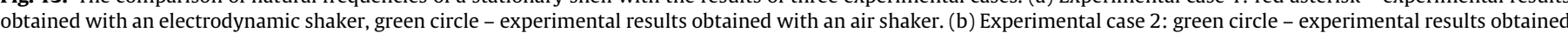

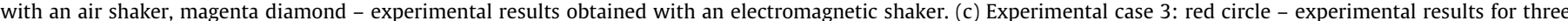

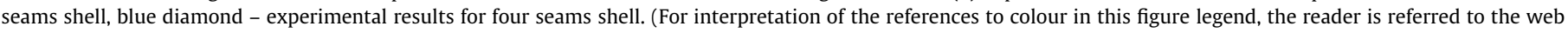
version of this article.)
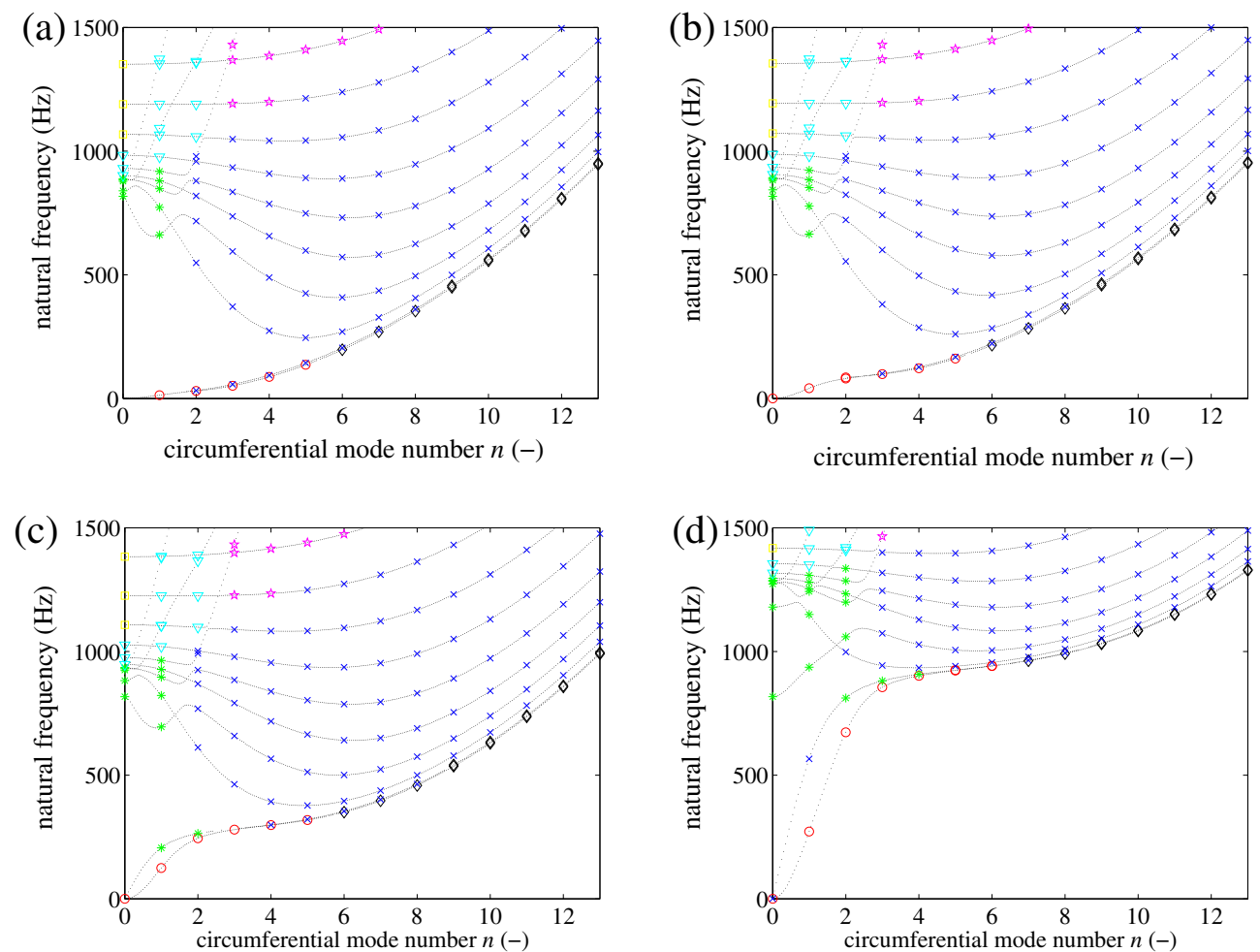

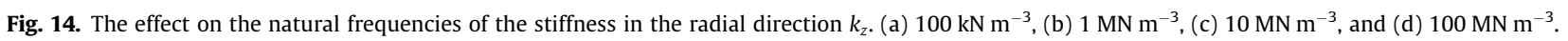




\section{Parametric study}

A parametric study is presented next in order to illustrate the effects of varying the radial and tangential stiffness of the elastic foundation, and the rotation speed of the shell.

In Fig. 14 the effect of the stiffness in the radial direction $k_{z}$ is shown. Here the same marker types as in Fig. 3 has been used to designate different root types. Four different stiffness values have been considered, from $100 \mathrm{kN} \mathrm{m}^{-3}$ to $100 \mathrm{MN} \mathrm{m}^{-3}$. The other properties are as given in Table 3. Results are shown for the frequency range from 0 to $1.5 \mathrm{kHz}$, and for the circumferential mode numbers from 0 to 13 .

As can be seen in Fig. 14 the natural frequencies of low-order modes generally increase when increasing the radial stiffness of the elastic foundation. The higher-order modes are less sensitive to the radial stiffness. This is a natural property of a stiffness constraint since its impedance decreases with frequency.

It is important to mention that a shell with free boundary conditions has 6 rigid body modes corresponding to zero eigenfrequencies. Four zero eigenfrequencies are shown in Fig. 3(a) in case of Rayleigh and Love branches with $n=0$ and $n=1$. The remaining two would correspond to Rayleigh and Love branches with $n=-1$. The physical meaning of $n=-1$, is discussed in the forthcoming part of the paper where the effects of rotation speed are considered.

Fig. 14 shows that as the elastic suspension stiffness increases, both Rayleigh and Love modes with $n=1$ gain a natural frequency. Thus the four out of six rigid body modes vanish and only two remain. These two that remain are the rotation around the $x$-axis and the translation along it, which are unaffected by radial spring constraints imposed. Their existence is clear in Fig. 14(c) and (d) where the two branches converge to zero frequency for $n=0$. It is also notable that at lower circumferential mode numbers the Rayleigh and Love branches are characterised by relatively large differences in natural frequencies, if a large radial suspension stiff-

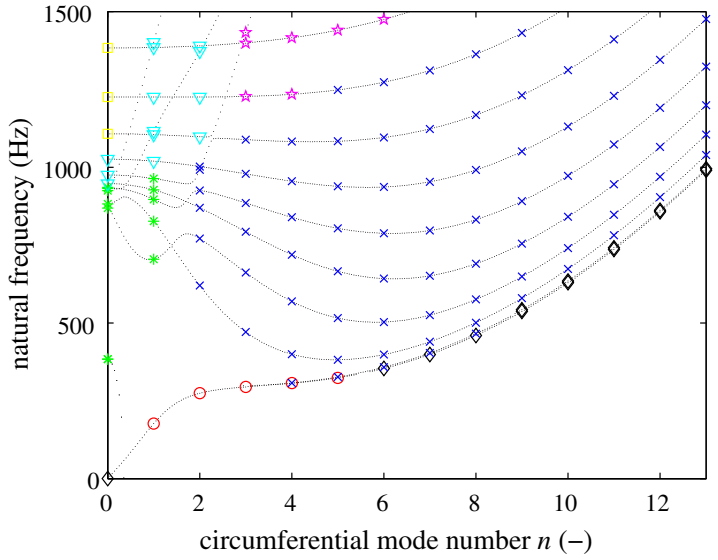

Fig. 16. The combined effect on the natural frequencies of the stiffness in the radial direction $k_{z}$ and the stiffness in the tangential direction $k_{\phi} .10 \mathrm{MN} \mathrm{m}^{-3}$ for both stiffness.

ness is imposed. This can be seen in Fig. 14 (d) since the two branches no longer overlap with $n=1-3$. This is because with Rayleigh $n=1$ modes the shell bounces on the radial spring with no nodal cross-sections (the shell axis remains parallel to the $x$-axis in Fig. 1), whereas with Love $n=1$ modes the shell rocks on the distributed radial spring rotating around the radial $z$-axis in Fig. 1. As such, the two $n=1$ modes are characterised by different modal stiffnesses/mass ratios imposed by the radial elastic suspension. However, as the $n$ number increases the two branches start to overlap again since the impedance of a pure stiffness vanishes with the increase of frequency.

Fig. 15 collects the effect of stiffness in the circumferential direction $k_{\phi}$ on the natural frequencies. Results for different stiffness values from $100 \mathrm{kN} \mathrm{m}^{-3}$ to $100 \mathrm{MN} \mathrm{m}^{-3}$ are presented. Also the same
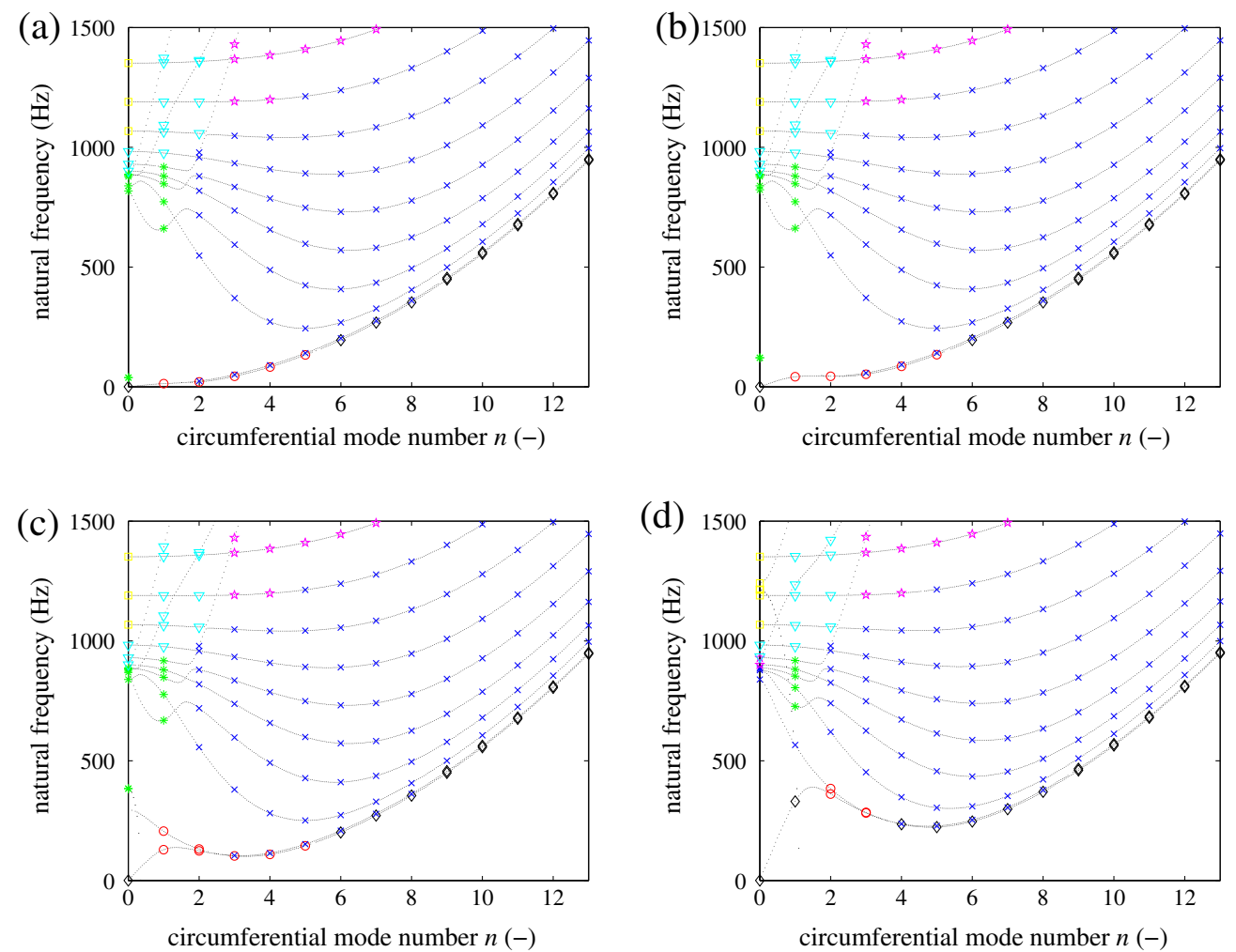

Fig. 15. The effect on the natural frequencies of the stiffness in the tangential direction $k_{\phi}$. (a) $100 \mathrm{kN} \mathrm{m}^{-3}$, (b) $1 \mathrm{MN} \mathrm{m}^{-3}$, (c) $10 \mathrm{MN} \mathrm{m}^{-3}$, and (d) $100 \mathrm{MN} \mathrm{m}^{-3}$. 

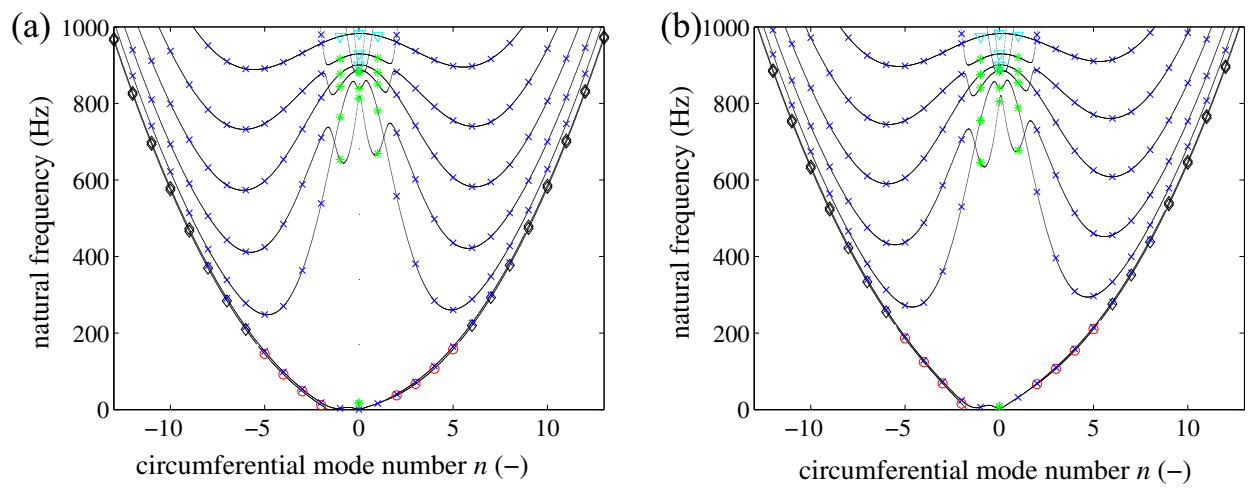

Fig. 17. The effect on the natural frequencies of the rotation speed $\Omega$. (a) $100 \mathrm{rad} / \mathrm{s}$ and (b) $200 \mathrm{rad} / \mathrm{s}$.

layout as in Fig. 3 has been used, and the frequency and the circumferential mode numbers range are the same as in Fig. 14. In this case it can be seen that the rigid body mode representing the rotation around the $x$-axis now gains a non-zero natural frequency as shown by the green asterisk in Fig. 15(a)-(c). This is because the tangentially stiff foundation couples with this type of motion. The $n=1$ Love mode also gains a natural frequency since the non-exact representations of the rigid body rocking of the shell, shown in Fig. 9 (a), and discussed earlier on, induce tangential displacement components. Therefore the result with $n=1$ Love mode is probably not accurate. Regarding now the Rayleigh branch, it goes to $0 \mathrm{~Hz}$ for $n=0$, which represents the rigid body translation along the $\mathrm{x}$ axis which is not sensitive to tangential foundation stiffness.

The combined effect of both the radial and the tangential stiffness has also been studied and collected in Fig. 16. The value assigned for both stiffness is $10 \mathrm{MNm}^{-3}$. It can be seen that when the shell is subject to stiffness in both directions, the only rigid body mode that remains is the translation along the $x$-axis (the black diamond in the origin of the plot).

The results for the rotating case are considered next, in Fig. 17, that follows the same layout as Fig. 3. Here the ordinate shows absolute values of natural frequencies. The fact that the natural frequencies can be mathematically negative is instead designated by a negative circumferential mode number. This way it is easier to compare the branches on the left hand side of the ordinate to those on the right hand side of the ordinate axis.

The plot (a) is for the constant rotation speed of $100 \mathrm{rad} / \mathrm{s}$, whereas the plot (b) is for the constant rotation speed of $200 \mathrm{rad} / \mathrm{s}$ around the $x$ - axis of the reference frame. It can be seen by comparing the two plots that, in general, the higher the rotation speed, the higher is the natural frequency of a particular mode. This is due to the effects of the hoop stress, which, as indicated in the Eq. (11) arises with the centrifugal forces. It can also be seen that the branches are no longer symmetric. Thus, the natural frequencies of the forward rotating modes (in the direction of rotation of the shell) are now different from their backward rotating counterparts, as they now rotate in opposite directions at different speeds (see Eqs. (12)-(14)) without a possibility to superimpose. It is interesting to note that the natural frequencies of the modes with $n=0$ do not veer with the rotation speed $\Omega$ since all branches in Fig. 17 are continuous at $n=0$ such that no two distinct absolute values for the two natural frequencies occur. Mathematically this could be shown by substituting $n=0$ into Eqs. (16)-(25) which would cause the odd coefficients of the characteristic polynomial written in terms of $\omega_{m, n}$ to vanish. Thus the forward and the backward rotating modes have the natural frequencies equal in their absolute value but opposite in sign, so the "regular" stationary modes occur with $n=0$ even if the shell spins. The even coefficients of order 2 and 4 would still contain rotation speed $\Omega$ so the natural frequency of these stationary modes would not be the same for the rotating and the stationary shells. Physically the modes with $n=0$ are characterised by the axial symmetry of the bending deformations. They are governed by the root types 6,7 , and 8 . They can be bending (Figs. 10(a) and 11(a)), longitudinal (Fig. 10(b) or shear modes (Figs. 11(b) and 12(a)).

\section{Conclusions}

An exact method is developed to calculate natural frequencies and mode shapes of a rotating cylindrical shell having free boundary conditions. Effects of the flexible support in the radial and circumferential direction, and the initial hoop stresses due to a possible pressurisation and/or centrifugal forces, are considered in the model. Eight types of roots of the characteristic polynomial equation are considered, and the corresponding mathematical expressions for eight types of mode shapes are derived. This is relevant for both rotating and non-rotating shells since in either situation it is shown that the axial profiles of modes are governed by a certain combination of circular and hyperbolic functions. This combination is determined by the root type. For each of the eight root types and the corresponding mode shape functions, the boundary conditions are satisfied exactly. Thus the model presented in this study may be considered to constitute a complete analytical solution for the free vibration problem of rotating cylindrical shells having free ends, which are supported by an elastic foundation. An excellent agreement between natural frequencies obtained in experimental studies and the present method is demonstrated for stationary shells. For a certain type of rigid body rocking mode, however, care must be taken with the assumed mode shape function and its influence on the obtained natural frequency in case of relatively short shells. Furthermore, it must be noted that the method developed, although exact, requires a rather complicated procedure of the undetermined shell length to perform the analysis. Thus, as a future work, an approximate Rayleigh-Ritz methodology utilising beam functions to approximate the mode shape axial profile will be considered.

\section{Acknowledgements}

This project has received funding from the European Union's Horizon 2020 research and innovation programme under the Marie Sklodowska-Curie grant agreement no. 657539. This work benefits from the Belgian Programme on Interuniversity Attraction Poles, initiated by the Belgian Federal Science Policy Office (DYSCO). The authors acknowledge the financial support from the COST Action TU1105. Also, the Research Fund KU Leuven is gratefully acknowledged for its support. Finally, the authors acknowledge the financial support from IWT Flanders within the MODRIO project. 


\section{Appendix A. Appendix}

\section{A.1. Case 1: two real, two imaginary and four complex roots}

The coefficients $t_{r, s, \sim}$ in case $\alpha_{r}= \pm \alpha_{1}, \pm \mathrm{i} \gamma_{2}, \pm(p \pm \mathrm{i} q)$ are given in Table A1.

\section{Table A1}

The coefficients $t_{r, s, \sim}$ in case $\alpha_{r}= \pm \alpha_{1}, \pm \mathrm{i} \gamma_{2}, \pm(p \pm \mathrm{i} q)$.

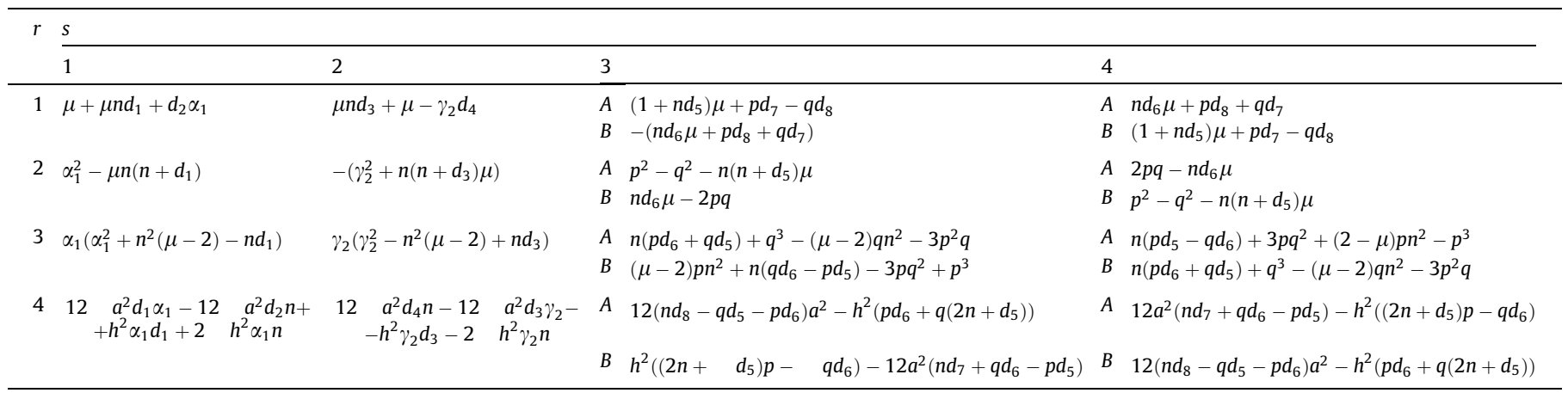

\section{A.2. Case 2: eight complex roots}

The coefficients $t_{r, s, \sim}$ in case $\alpha_{r}=\{ \pm(f \pm \mathrm{i} g), \pm(p \pm \mathrm{i} q)\}$ are given in Table A2.

\section{Table A2}

The coefficients $t_{r, s, \sim}$ in case $\alpha_{r}= \pm(f \pm \mathrm{i} g), \pm(p \pm \mathrm{i} q)$.

\begin{tabular}{|c|c|c|c|c|c|c|c|c|}
\hline \multirow[t]{2}{*}{$r$} & \multicolumn{8}{|l|}{$S$} \\
\hline & 1 & & 2 & & 3 & & 4 & \\
\hline 1 & $\begin{array}{l}A \\
B\end{array}$ & $\begin{array}{l}\mu d_{1} n+\mu+p d_{3}-q d_{4} \\
-d_{2} n \mu-p d_{4}-q d_{3}\end{array}$ & $\begin{array}{l}A \\
B\end{array}$ & $\begin{array}{l}d_{2} n \mu+p d_{4}+q d_{3} \\
\mu d_{1} n+\mu+p d_{3}-q d_{4}\end{array}$ & $\begin{array}{l}A \\
B\end{array}$ & $\begin{array}{l}\mu d_{5} n+\mu-g d_{8}+f d_{7} \\
-d_{6} n \mu-f d_{8}-g d_{7}\end{array}$ & $\begin{array}{l}A \\
B\end{array}$ & $\begin{array}{l}d_{6} n \mu+f d_{8}+g d_{7} \\
\mu d_{5} n+\mu-g d_{8}+f d_{7}\end{array}$ \\
\hline 2 & $\begin{array}{l}A \\
B\end{array}$ & $\begin{array}{l}p^{2}-q^{2}-n^{2} \mu-\mu d_{1} n \\
d_{2} n \mu-2 p q\end{array}$ & $\begin{array}{l}A \\
B\end{array}$ & $\begin{array}{l}2 p q-d_{2} n \mu \\
p^{2}-q^{2}-n^{2} \mu-\mu d_{1} n\end{array}$ & $\begin{array}{l}A \\
B\end{array}$ & $\begin{array}{l}-n^{2} \mu-\mu d_{5} n+f^{2}-g^{2} \\
d_{6} n \mu-2 f g\end{array}$ & $\begin{array}{l}A \\
B\end{array}$ & $\begin{array}{l}2 f g-d_{6} n \mu \\
-n^{2} \mu-\mu d_{5} n+f^{2}-g^{2}\end{array}$ \\
\hline 3 & $A$ & $\begin{array}{l}-q n^{2} \mu+2 q n^{2}+n d_{1} q+ \\
\quad+n d_{2} p-3 p^{2} q+q^{3} \\
p n^{2} \mu-2 p n^{2}-n p d_{1}+ \\
\quad+n q d_{2}-3 p q^{2}+p^{3}\end{array}$ & $A$ & $\begin{array}{l}p n^{2} \mu-2 p n^{2}-n p d_{1}+ \\
+n q d_{2}-3 p q^{2}+p^{3} \\
q n^{2} \mu-2 q n^{2}-n d_{1} q- \\
-n d_{2} p+3 p^{2} q-q^{3}\end{array}$ & $A$ & $\begin{array}{l}-g n^{2} \mu+2 g n^{2}+n f d_{6}+ \\
+n g d_{5}-3 f^{2} g+g^{3} \\
f n^{2} \mu-2 f n^{2}+n d_{6} g- \\
-n d_{5} f+f^{3}-3 f g^{2}\end{array}$ & $A$ & $\begin{array}{l}f n^{2} \mu-2 f n^{2}+n d_{6} g- \\
-n d_{5} f+f^{3}-3 f g^{2} \\
g n^{2} \mu-2 g n^{2}-n f d_{6}- \\
-n g d_{5}+3 f^{2} g-g^{3}\end{array}$ \\
\hline 4 & $A$ & $\begin{array}{l}a^{2}\left(d_{4} n-d_{1} q-d_{2} p\right)-1 / 6 h^{2} q n- \\
\quad-1 / 12 h^{2} d_{2} p-1 / 12 h^{2} d_{1} q \\
a^{2}\left(p d_{1}-q d_{2}-d_{3} n\right)+1 / 6 h^{2} p n+ \\
\quad+1 / 12 h^{2} p d_{1}-1 / 12 h^{2} q d_{2}\end{array}$ & $A$ & $\begin{array}{l}a^{2}\left(p d_{1}-q d_{2}-d_{3} n\right)+1 / 6 h^{2} p n+ \\
\quad+1 / 12 h^{2} p d_{1}-1 / 12 h^{2} q d_{2} \\
a^{2}\left(d_{1} q-d_{4} n+d_{2} p\right)+1 / 6 h^{2} q n+ \\
\quad+1 / 12 h^{2} d_{2} p+1 / 12 h^{2} d_{1} q\end{array}$ & $B$ & $\begin{array}{l}a^{2}\left(d_{8} n-f d_{6}-g d_{5}\right)-1 / 6 h^{2} g n- \\
\quad-1 / 12 h^{2} f d_{6}-1 / 12 h^{2} g d_{5} \\
a^{2}\left(d_{5} f-d_{7} n-d_{6} g\right)+1 / 6 h^{2} f n+ \\
\quad+1 / 12 h^{2} d_{5} f-1 / 12 h^{2} d_{6} g\end{array}$ & $B$ & $\begin{array}{l}a^{2}\left(d_{5} f-d_{7} n-d_{6} g\right)+1 / 6 h^{2} f n+ \\
\quad+1 / 12 h^{2} d_{5} f-1 / 12 h^{2} d_{6} g \\
a^{2} f d_{6}-a^{2} d_{8} n+a^{2} g d_{5}+1 / 6 h^{2} g n+ \\
\quad+1 / 12 h^{2} f d_{6}+1 / 12 h^{2} g d_{5}\end{array}$ \\
\hline
\end{tabular}

\section{A.3. Case 3: four real and four complex roots}

The coefficients $t_{r, s, \sim}$ in case $\alpha_{r}=\left\{ \pm \alpha_{1}, \pm \alpha_{2}, \pm(p \pm \mathrm{i} q)\right\}$ are given in Table A3.

\section{Table A3}

The coefficients $t_{r, s, \sim}$ in case $\alpha_{r}=\left\{ \pm \alpha_{1}, \pm \alpha_{2}, \pm(p \pm \mathrm{i} q)\right\}$.

\begin{tabular}{|c|c|c|c|c|c|c|}
\hline \multirow[t]{2}{*}{$r$} & \multicolumn{6}{|l|}{$s$} \\
\hline & 1 & 2 & 3 & & 4 & \\
\hline 1 & $\mu+\mu n d_{1}+d_{2} \alpha_{1}$ & $\alpha_{2} d_{4}+\mu n d_{3}+\mu$ & $\begin{array}{l}A \\
B\end{array}$ & $\begin{array}{l}\left(1+n d_{5}\right) \mu+p d_{7}-q d_{8} \\
-\left(n d_{6} \mu+p d_{8}+q d_{7}\right)\end{array}$ & $\begin{array}{l}A \\
B\end{array}$ & $\begin{array}{l}n d_{6} \mu+p d_{8}+q d_{7} \\
\left(1+n d_{5}\right) \mu+p d_{7}-q d_{8}\end{array}$ \\
\hline 2 & $\alpha_{1}^{2}-\mu n\left(n+d_{1}\right)$ & $-n\left(n+d_{3}\right) \mu+\alpha_{2}^{2}$ & $\begin{array}{l}A \\
B\end{array}$ & $\begin{array}{l}p^{2}-q^{2}-n\left(n+d_{5}\right) \mu \\
n d_{6} \mu-2 p q\end{array}$ & $\begin{array}{l}A \\
B\end{array}$ & $\begin{array}{l}2 p q-n d_{6} \mu \\
p^{2}-q^{2}-n\left(n+d_{5}\right) \mu\end{array}$ \\
\hline 3 & $\alpha_{1}\left(\alpha_{1}^{2}+n^{2}(\mu-2)-n d_{1}\right)$ & $-\alpha_{2}\left((2-\mu) n^{2}+n d_{3}-\alpha_{2}^{2}\right)$ & $\begin{array}{l}A \\
B\end{array}$ & $\begin{array}{l}n\left(p d_{6}+q d_{5}\right)+q^{3}-(\mu-2) q n^{2}-3 p^{2} q \\
(\mu-2) p n^{2}+n\left(q d_{6}-p d_{5}\right)-3 p q^{2}+p^{3}\end{array}$ & $\begin{array}{l}A \\
B\end{array}$ & $\begin{array}{l}n\left(p d_{5}-q d_{6}\right)+3 p q^{2}+(2-\mu) p n^{2}-p^{3} \\
n\left(p d_{6}+q d_{5}\right)+q^{3}-(\mu-2) q n^{2}-3 p^{2} q\end{array}$ \\
\hline 4 & $\begin{array}{l}a^{2}\left(d_{1} \alpha_{1}-d_{2} n\right)+ \\
+h^{2} \alpha_{1}\left(1 / 12 \quad d_{1}+1 / 6 \quad n\right)\end{array}$ & $\begin{array}{l}a^{2}\left(d_{3} \alpha_{2}-n d_{4}\right)+ \\
+1 / 12 h^{2} \alpha_{2}\left(2 n+d_{3}\right)\end{array}$ & $A$ & $\begin{array}{l}\left(n d_{8}-q d_{5}-p d_{6}\right) a^{2}+ \\
-\left(1 / 12 p d_{6}+1 / 12 q d_{5}+1 / 6 q n\right) h^{2} \\
\left(p d_{5}-n d_{7}-q d_{6}\right) a^{2}+ \\
\left(1 / 6 n p+1 / 12 p d_{5}-1 / 12 q d_{6}\right) h^{2}\end{array}$ & $B$ & $\begin{array}{l}\left(p d_{5}-n d_{7}-q d_{6}\right) a^{2}+ \\
\left(1 / 6 n p+1 / 12 p d_{5}-1 / 12 q d_{6}\right) h^{2} \\
\left(1 / 12 p d_{6}+1 / 12 q d_{5}+1 / 6 q n\right) h^{2}- \\
-\left(n d_{8}-q d_{5}-p d_{6}\right) a^{2}\end{array}$ \\
\hline
\end{tabular}




\section{A.4. Case 4: eight real roots}

The coefficients $t_{r, s, \sim}$ in case $\alpha_{r}=\left\{ \pm \alpha_{1}, \pm \alpha_{2}, \pm \alpha_{3}, \pm \alpha_{4}\right\}$ are given in Table A4.

Table A4

The coefficients $t_{r, s, \sim}$ in case $\alpha_{r}=\left\{ \pm \alpha_{1}, \pm \alpha_{2}, \pm \alpha_{3}, \pm \alpha_{4}\right\}$.

\begin{tabular}{|c|c|c|c|c|c|}
\hline \multirow[t]{2}{*}{$r$} & \multicolumn{5}{|l|}{$s$} \\
\hline & 1 & & 2 & 3 & 4 \\
\hline 1 & $\mu+\mu n d_{1}+d_{2} \alpha_{1}$ & & $\alpha_{2} d_{4}+\mu n d_{3}+\mu$ & $\left(1+n d_{5}\right) \mu+\alpha_{3} d_{6}$ & $\mu n d_{7}+\mu+\alpha_{4} d_{8}$ \\
\hline 2 & $\alpha_{1}^{2}-\mu n\left(n+d_{1}\right)$ & & $-n\left(n+d_{3}\right) \mu+\alpha_{2}^{2}$ & $-n\left(n+d_{5}\right) \mu+\alpha_{3}^{2}$ & $-n\left(n+d_{7}\right) \mu+\alpha_{4}^{2}$ \\
\hline 3 & $\alpha_{1}\left(\alpha_{1}^{2}+n^{2}(\mu-2)-n d_{1}\right)$ & & $-\alpha_{2}\left((2-\mu) n^{2}+n d_{3}-\alpha_{2}^{2}\right)$ & $-\alpha_{3}\left((2-\mu) n^{2}+n d_{5}-\alpha_{3}^{2}\right)$ & $-\left((2-\mu) n^{2}+n d_{7}-\alpha_{4}^{2}\right) \alpha_{4}$ \\
\hline 4 & $a^{2}\left(d_{1} \alpha_{1}-d_{2} n\right)+h^{2} \alpha_{1}(1 / 12$ & $\left.d_{1}+1 / 6 \quad n\right)$ & $a^{2}\left(d_{3} \alpha_{2}-n d_{4}\right)+1 / 12 h^{2} \alpha_{2}\left(2 n+d_{3}\right)$ & $\left(d_{5} \alpha_{3}-d_{6} n\right) a^{2}+1 / 12 h^{2} \alpha_{3}\left(d_{5}+2 n\right)$ & $\left(d_{7} \alpha_{4}-d_{8} n\right) a^{2}+1 / 12 h^{2} \alpha_{4}\left(d_{7}+2 n\right)$ \\
\hline
\end{tabular}

\section{A.5. Case 5: six real and two imaginary roots}

The coefficients $t_{r, s, \sim}$ in case $\alpha_{r}=\left\{ \pm \alpha_{1}, \pm \alpha_{2}, \pm \alpha_{3}, \pm \mathrm{i} \gamma_{4}\right\}$ are given in Table A5.

\section{Table A5}

The coefficients $t_{r, s, \sim}$ in case $\alpha_{r}=\left\{ \pm \alpha_{1}, \pm \alpha_{2}, \pm \alpha_{3}, \pm \mathrm{i} \gamma_{4}\right\}$.

\begin{tabular}{|c|c|c|c|c|}
\hline \multirow[t]{2}{*}{$r$} & \multicolumn{4}{|l|}{$S$} \\
\hline & 1 & 2 & 3 & 4 \\
\hline 1 & $\mu+\mu n d_{1}+d_{2} \alpha_{1}$ & $\alpha_{2} d_{4}+\mu n d_{3}+\mu$ & $\left(1+n d_{5}\right) \mu+\alpha_{3} d_{6}$ & $\mu n d_{7}+\mu-\gamma_{4} d_{8}$ \\
\hline 2 & $\alpha_{1}^{2}-\mu n\left(n+d_{1}\right)$ & $-n\left(n+d_{3}\right) \mu+\alpha_{2}^{2}$ & $-n\left(n+d_{5}\right) \mu+\alpha_{3}^{2}$ & $-n\left(n+d_{7}\right) \mu-\gamma_{4}^{2}$ \\
\hline 3 & $\alpha_{1}\left(\alpha_{1}^{2}+n^{2}(\mu-2)-n d_{1}\right)$ & $-\alpha_{2}\left((2-\mu) n^{2}+n d_{3}-\alpha_{2}^{2}\right)$ & $-\alpha_{3}\left((2-\mu) n^{2}+n d_{5}-\alpha_{3}^{2}\right)$ & $\left((2-\mu) n^{2}+n d_{7}+\gamma_{4}^{2}\right) \gamma_{4}$ \\
\hline 4 & $a^{2}\left(d_{1} \alpha_{1}-d_{2} n\right)+h^{2} \alpha_{1}\left(1 / 12 \quad d_{1}+1 / 6 \quad n\right)$ & $a^{2}\left(d_{3} \alpha_{2}-n d_{4}\right)+1 / 12 h^{2} \alpha_{2}\left(2 n+d_{3}\right)$ & $\left(d_{5} \alpha_{3}-d_{6} n\right) a^{2}+1 / 12 h^{2} \alpha_{3}\left(d_{5}+2 n\right)$ & $\left(-d_{7} \gamma_{4}+d_{8} n\right) a^{2}-1 / 12 h^{2} \gamma_{4}\left(d_{7}+2 n\right)$ \\
\hline
\end{tabular}

\section{A.6. Case 6: four real and four imaginary roots}

The coefficients $t_{r, s, \sim}$ in case $\alpha_{r}=\left\{ \pm \alpha_{1}, \pm \alpha_{2}, \pm \mathrm{i} \gamma_{3}, \pm \mathrm{i} \gamma_{4}\right\}$ are given in Table A6.

Table A6

The coefficients $t_{r, s, \sim}$ in case $\alpha_{r}=\left\{ \pm \alpha_{1}, \pm \alpha_{2}, \pm \mathrm{i} \gamma_{3}, \pm \mathrm{i} \gamma_{4}\right\}$.

\begin{tabular}{|c|c|c|c|c|c|}
\hline \multirow[t]{2}{*}{$r$} & \multicolumn{5}{|l|}{$s$} \\
\hline & 1 & & 2 & 3 & 4 \\
\hline 1 & $\mu+\mu n d_{1}+d_{2} \alpha_{1}$ & & $\alpha_{2} d_{4}+\mu n d_{3}+\mu$ & $\left(1+n d_{5}\right) \mu-\gamma_{3} d_{6}$ & $\mu n d_{7}+\mu-\gamma_{4} d_{8}$ \\
\hline 2 & $\alpha_{1}^{2}-\mu n\left(n+d_{1}\right)$ & & $-n\left(n+d_{3}\right) \mu+\alpha_{2}^{2}$ & $-n\left(n+d_{5}\right) \mu-\gamma_{3}^{2}$ & $-n\left(n+d_{7}\right) \mu-\gamma_{4}^{2}$ \\
\hline 3 & $\alpha_{1}\left(\alpha_{1}^{2}+n^{2}(\mu-2)-n d_{1}\right)$ & & $-\alpha_{2}\left((2-\mu) n^{2}+n d_{3}-\alpha_{2}^{2}\right)$ & $\left((2-\mu) n^{2}+n d_{5}+\gamma_{3}^{2}\right) \gamma_{3}$ & $\left((2-\mu) n^{2}+n d_{7}+\gamma_{4}^{2}\right) \gamma_{4}$ \\
\hline 4 & $a^{2}\left(d_{1} \alpha_{1}-d_{2} n\right)+h^{2} \alpha_{1}(1 / 12$ & $\left.d_{1}+1 / 6 \quad n\right)$ & $a^{2}\left(d_{3} \alpha_{2}-n d_{4}\right)+1 / 12 h^{2} \alpha_{2}\left(2 n+d_{3}\right)$ & $-\left(d_{5} \gamma_{3}-d_{6} n\right) a^{2}-1 / 12 h^{2} \gamma_{3}\left(d_{5}+2 n\right)$ & $\left(-d_{7} \gamma_{4}+d_{8} n\right) a^{2}-1 / 12 h^{2} \gamma_{4}\left(d_{7}+2 n\right)$ \\
\hline
\end{tabular}

\section{A.7. Case 7: two real and six imaginary roots}

The coefficients $t_{r, s, \sim}$ in case $\alpha_{r}=\left\{ \pm \alpha_{1}, \pm \mathbf{i} \gamma_{2}, \pm \mathbf{i} \gamma_{3}, \pm \mathbf{i} \gamma_{4}\right\}$ are given in Table A7.

\section{Table A7}

The coefficients $t_{r, s, \sim}$ in case $\alpha_{r}=\left\{ \pm \alpha_{1}, \pm \mathrm{i} \gamma_{2}, \pm \mathrm{i} \gamma_{3}, \pm \mathrm{i} \gamma_{4}\right\}$.

\begin{tabular}{|c|c|c|c|c|c|}
\hline \multirow[t]{2}{*}{$r$} & \multicolumn{5}{|l|}{$s$} \\
\hline & 1 & & 2 & 3 & 4 \\
\hline 1 & $\mu+\mu n d_{1}+d_{2} \alpha_{1}$ & & $-\gamma_{2} d_{4}+\mu n d_{3}+\mu$ & $\left(1+n d_{5}\right) \mu-\gamma_{3} d_{6}$ & $\mu n d_{7}+\mu-\gamma_{4} d_{8}$ \\
\hline 2 & $\alpha_{1}^{2}-\mu n\left(n+d_{1}\right)$ & & $-n\left(n+d_{3}\right) \mu+\gamma_{2}^{2}$ & $-n\left(n+d_{5}\right) \mu-\gamma_{3}^{2}$ & $-n\left(n+d_{7}\right) \mu-\gamma_{4}^{2}$ \\
\hline 3 & $\alpha_{1}\left(\alpha_{1}^{2}+n^{2}(\mu-2)-n d_{1}\right)$ & & $\gamma_{2}\left((2-\mu) n^{2}+n d_{3}+\gamma_{2}^{2}\right)$ & $\left((2-\mu) n^{2}+n d_{5}+\gamma_{3}^{2}\right) \gamma_{3}$ & $\left((2-\mu) n^{2}+n d_{7}+\gamma_{4}^{2}\right) \gamma_{4}$ \\
\hline 4 & $a^{2}\left(d_{1} \alpha_{1}-d_{2} n\right)+h^{2} \alpha_{1}(1 / 12$ & $\left.d_{1}+1 / 6 \quad n\right)$ & $-a^{2}\left(d_{3} \gamma_{2}-n d_{4}\right)-1 / 12 h^{2} \gamma_{2}\left(2 n+d_{3}\right)$ & $-\left(d_{5} \gamma_{3}-d_{6} n\right) a^{2}-1 / 12 h^{2} \gamma_{3}\left(d_{5}+2 n\right)$ & $\left(-d_{7} \gamma_{4}+d_{8} n\right) a^{2}-1 / 12 h^{2} \gamma_{4}\left(d_{7}+2 n\right)$ \\
\hline
\end{tabular}




\section{A.8. Case 8: four imaginary and four complex roots}

The coefficients $t_{r, s, \sim}$ in case $\alpha_{r}=\left\{ \pm \mathrm{i} \gamma_{1}, \pm \mathrm{i} \gamma_{2}, \pm(p \pm \mathrm{i} q)\right\}$ are (see

Table A8):

Table A8

The coefficients $t_{r, s, \sim}$ in case $\alpha_{r}=\left\{ \pm \mathrm{i} \gamma_{1}, \pm \mathrm{i} \gamma_{2}, \pm(p \pm \mathrm{i} q)\right\}$.

\begin{tabular}{|c|c|c|c|c|c|c|}
\hline \multirow[t]{2}{*}{$r$} & \multicolumn{6}{|l|}{$S$} \\
\hline & 1 & 2 & 3 & & 4 & \\
\hline 1 & $\left(1+n d_{1}\right) \mu-\gamma_{1} d_{2}$ & $-\gamma_{2} d_{4}+\mu n d_{3}+\mu-n\left(n+d_{3}\right) \mu+\gamma_{2}^{2}$ & $\begin{array}{l}A \\
B\end{array}$ & $\begin{array}{l}\left(1+n d_{5}\right) \mu+p d_{7}-q d_{8} \\
-\left(n d_{6} \mu+p d_{8}+q d_{7}\right)\end{array}$ & $\begin{array}{l}A \\
B\end{array}$ & $\begin{array}{l}n d_{6} \mu+p d_{8}+q d_{7} \\
\left(1+n d_{5}\right) \mu+p d_{7}-q d_{8}\end{array}$ \\
\hline 2 & $-n\left(n+d_{1}\right) \mu-\gamma_{1}^{2}$ & $\gamma_{2}\left((2-\mu) n^{2}+n d_{3}+\gamma_{2}^{2}\right)$ & $\begin{array}{l}A \\
B\end{array}$ & $\begin{array}{l}p^{2}-q^{2}-n\left(n+d_{5}\right) \mu \\
n d_{6} \mu-2 p q\end{array}$ & $\begin{array}{l}A \\
B\end{array}$ & $\begin{array}{l}2 p q-n d_{6} \mu \\
p^{2}-q^{2}-n\left(n+d_{5}\right) \mu\end{array}$ \\
\hline 3 & $\gamma_{1}\left((2-\mu) n^{2}+n d_{1}+\gamma_{1}^{2}\right)$ & $-\gamma_{2} d_{4}+\mu n d_{3}+\mu-n\left(n+d_{3}\right) \mu+\gamma_{2}^{2}$ & $\begin{array}{l}A \\
B\end{array}$ & $\begin{array}{l}n\left(p d_{6}+q d_{5}\right)+q^{3}-(\mu-2) q n^{2}-3 p^{2} q \\
(\mu-2) p n^{2}+n\left(q d_{6}-p d_{5}\right)-3 p q^{2}+p^{3}\end{array}$ & $\begin{array}{l}A \\
B\end{array}$ & $\begin{array}{l}n\left(p d_{5}-q d_{6}\right)+3 p q^{2}+(2-\mu) p n^{2}-p^{3} \\
n\left(p d_{6}+q d_{5}\right)+q^{3}-(\mu-2) q n^{2}-3 p^{2} q\end{array}$ \\
\hline 4 & $-\left(d_{1} \gamma_{1}-d_{2} n\right) a^{2}-1 / 12 h^{2} \gamma_{1}\left(d_{1}+2 n\right)$ & $\gamma_{2}\left((2-\mu) n^{2}+n d_{3}+\gamma_{2}^{2}\right)$ & $A$ & $\begin{array}{l}\left(n d_{8}-q d_{5}-p d_{6}\right) a^{2}+ \\
-\left(1 / 12 p d_{6}+1 / 12 q d_{5}+1 / 6 q n\right) h^{2} \\
\left(p d_{5}-n d_{7}-q d_{6}\right) a^{2}+ \\
\left(1 / 6 n p+1 / 12 p d_{5}-1 / 12 q d_{6}\right) h^{2}\end{array}$ & $A$ & $\begin{array}{l}\left(p d_{5}-n d_{7}-q d_{6}\right) a^{2}+ \\
\left(1 / 6 n p+1 / 12 p d_{5}-1 / 12 q d_{6}\right) h^{2} \\
\left(1 / 12 p d_{6}+1 / 12 q d_{5}+1 / 6 q n\right) h^{2}- \\
-\left(n d_{8}-q d_{5}-p d_{6}\right) a^{2}\end{array}$ \\
\hline
\end{tabular}

All the coefficients $t_{r, s, \sim}$ as well as $b_{r}$, together with the sixteen determinants can be found in Ref. [41].

\section{References}

[1] Poisson SD. Sur l'équilibre et le mouvement des corps élastiques. (On the equilibrium and motion of elastic bodies). Paris: Memoirs of the Paris Academy; 1829.

[2] Kirchhoff GR. Über das Gleichgewicht und die Bewegung einer elastischen Scheibe. (On the equilibrium and motion of an elastic disc). J Math (Crelle) $1850 ; 40$.

[3] Aron H. Das Gleichgewicht und die Bewegung einer unendlich dünnen, beliebig gekrümmten elastischen Schale. (The equilibrium and the motion of an infinitely thin, arbitrarily curved elastic shell). J Math (Crelle) 1874;78.

[4] Love AEH. On the small free vibrations and deformations of thin elastic shells. Philos Trans Roy Soc London 1888;179A.

[5] Rayleigh JWS. On the infinitesimal bending of surfaces of revolution. Math Soc Proc 13, London 1882.

[6] Novozhilov VV. The theory of thin elastic shells. Gromingen, The Netherlands: P. Noordhoff; 1964.

[7] Flügge W. Statik and Dynamik der Schalen (Statics and dynamics of shells). Berlin: Springer-Verlag; 1934.

[8] Byrne R. Theory of small deformations of a thin elastic shell. Sem Rep Math Univ Calif Publ Math, NS 1944;2(1):103-52.

[9] Goldenveizer AL. Theory of thin shells. Elmsford, NY: Pergamon Press; 1961.

[10] Naghdi PM, Berry JG. On the equations of motion of cylindrical shells. J App. Mech 1964;21(2):160-6.

[11] Lur'ye AI. General theory of elastic shells. Prikl Mat Meh 1940;4(1):7-34 [in Russian].

[12] Sanders JL. An improved first approximation theory for thin shells, NASA TRR24. Washington, DC: U.S. Government Printing Office; 1959.

[13] Reissner E. A new derivation of the equations for the deformation of elastic shells. Am J Math 1941:63:177-84.

[14] Vlasov VZ. General theory of shells and its applications in engineering (translation from Russian), NASA TTF-99. Washington (DC): U.S. Governmental Printing Office; 1964.

[15] Donnell LH. Beams, plates, and shells. New York: McGraw-Hill; 1976.

[16] Timoshenko S. Vibration problems in engineering. Princeton (NJ): D. Van Nostrand; 1955.

[17] Mushtari KM. Certain generalizations of the theory of thin shells. Izv Fiz Mat Kaz 1938;11(8)

[18] Leissa AW. Vibrations of shells. NASA SP-288. Washington (DC): U.S. Government Printing Office; 1973.

[19] Soedel W. Vibrations of shells and plates. 3rd ed. NewYork: Marcel Dekker Inc.; 2004 [revised and expanded].

[20] Bryan GH. On the beats in the vibrations of a revolving cylinder or bell. In: Proceedings of the Cambridge philosophical society; 1880. p. 101-11.

[21] Di Taranto RA, Lessen M. Coriolis acceleration effect on the vibration of a rotating thin-walled circular cylinder. J Appl Mech 1964;31:700-1.
[22] Srinivasan AV, Lauterbach GF. Traveling waves in rotating cylindrical shells. J Eng Ind ASME 1971;93:1229-32.

[23] Zohar A, Aboudi J. The free vibrations of a thin circular finite rotating cylinder. Int J Mech Sci 1973;15:269-78.

[24] Saito T, Endo M. Vibration of finite length, rotating cylindrical shells. J Sound Vib 1986;107:17-28.

[25] Endo M, Hatamura K, Sakata M, Taniguchi O. Flexural vibration of a thin rotating ring. J Sound Vib 1984;92:261-72.

[26] Padovan J. Natural frequencies of rotating prestressed cylinders. J Sound Vib 1973;31:469-82.

[27] Kim Y-J, Bolton JS. Effects of rotation on the dynamics of a circular cylindrical shell with application to tire vibration. J Sound Vib 2004;275:605-21.

[28] Huang SC, Soedel W. On the forced vibration of simply supported rotating cylindrical shells. J Acoust Soc Am 1988;84(1):275-85.

[29] Herrmann G, Armenakas AE. Dynamic behavior of cylindrical shells under initial stress. In: Proc 4th US nat congr appl mech ASME; 1962. p. 203-13.

[30] Molisani LR, Burdisso RA, Tsihlas D. A coupled tire structure/acoustic cavity model. Int J Solids Struct 2003;40:5125-38.

[31] Yu YY. Free vibrations of thin cylindrical shells having finite lengths with freely supported and clamped edges. J Appl Mech 1955;22(4).

[32] Donnell LH. A discussion of thin shell theory. In: Proceedings of the fifth international congress of applied mechanics; 1938.

[33] Vlasov VZ. Basic differential equations in the general theory of elastic shells. NACA TM 1241 (translated from 1944 Russian version); 1951.

[34] Forsberg K. Influence of boundary conditions on the modal characteristics of thin cylindrical shells. AIAA J 1964;2(12):2150-7.

[35] Weingarten VI. On the free vibration of thin cylindrical shells. Aerospace corporation, Systems research and planning division, El Segundo, California, Report No. TDR.169(3560.30)TN-3; December 1962

[36] Warburton GB. Vibration of thin cylindrical shells. J Mech Eng Sci 1965;7(4).

[37] Chung H. Free vibration analysis of circular cylindrical shells. J Sound Vib 1981:74:331-50.

[38] Sun S, Chu S, Cao D. Vibration characteristics of thin rotating cylindrical shells with various boundary conditions. J Sound Vib 2012;331:4170-86.

[39] Sun S, Cao D, Han Q. Vibration studies of rotating cylindrical shells with arbitrary edges using characteristic orthogonal polynomials in the RayleighRitz method. Int J Mech Sci 2013;68:180-9.

[40] Hu WCL, Wah T. Vibrations of ring-stiffened cylindrical shells - an "Exact" Method. Tech. Rept. No. 7, Contract NASr-94(06), SWRI Proj. 02-1504, Southwest Research Institute; October 1966.

[41] Alujevic N, Campillo-Davo N. Coefficients for calculating resonance frequencies of a rotating cylindrical shell having free boundary conditions, Free access: <https://www.fsb.unizg.hr/starmas/coefficients.txt>.

[42] Sewall JL, Naumann EC. An experimental and analytical vibration study of thin cylindrical shells with and without longitudinal stiffeners. Langley Research Center, NASA Technical Note, NASA TN D-4705; September 1968.

[43] Watkins JD, Clary R. Vibrational characteristics of some thin-walled cylindrical and conical frustum shells. Langley Research Center, NASA Technical Note, NASA TN D-2729; March 1965. 